ESAIM: M2AN 47 (2013) 689-715

DOI: $10.1051 / \mathrm{m} 2 \mathrm{an} / 2012044$
ESAIM: Mathematical Modelling and Numerical Analysis

www.esaim-m2an.org

\title{
A PIECEWISE $P_{2}$-NONCONFORMING QUADRILATERAL FINITE ELEMENT
}

\author{
Imbunm Kim ${ }^{1}$, Zhongxuan Luo ${ }^{2}$, Zhaoliang Meng ${ }^{2}, \mathrm{Hyun} \mathrm{Nam}^{3}$, Chunjae Park \\ AND DONGWOO SHEEN ${ }^{1,3}$
}

\begin{abstract}
We introduce a piecewise $P_{2}$-nonconforming quadrilateral finite element. First, we decompose a convex quadrilateral into the union of four triangles divided by its diagonals. Then the finite element space is defined by the set of all piecewise $P_{2}$-polynomials that are quadratic in each triangle and continuously differentiable on the quadrilateral. The degrees of freedom (DOFs) are defined by the eight values at the two Gauss points on each of the four edges plus the value at the intersection of the diagonals. Due to the existence of one linear relation among the above DOFs, it turns out the DOFs are eight. Global basis functions are defined in three types: vertex-wise, edge-wise, and element-wise types. The corresponding dimensions are counted for both Dirichlet and Neumann types of elliptic problems. For second-order elliptic problems and the Stokes problem, the local and global interpolation operators are defined. Also error estimates of optimal order are given in both broken energy and $L^{2}(\Omega)$ norms. The proposed element is also suitable to solve Stokes equations. The element is applied to approximate each component of velocity fields while the discontinuous $P_{1}$-nonconforming quadrilateral element is adopted to approximate the pressure. An optimal error estimate in energy norm is derived. Numerical results are shown to confirm the optimality of the presented piecewise $P_{2}$-nonconforming element on quadrilaterals.
\end{abstract}

Mathematics Subject Classification. 65N30, 76M10.

Received January 3, 2012. Revised July 16, 2012.

Published online March 4, 2013.

\section{INTRODUCTION}

It has been well-known that the use of standard lowest order conforming elements in solving solid and fluid mechanics problems produces undesirable unstable numerical solutions $[4,5,8,13,19,32]$. In order to avoid these numerical locking and checker-board solutions, engineers and scientists have developed and used alternatively higher-order conforming elements [35], techniques to stabilize the finite element method by adding suitable bubble functions [2] or modify the variational forms by adding stabilization terms $[12,15,26,31,36]$. Indeed, Pierre [47,48], Bank-Welfert [6], and Brezzi et al. [10] observed equivalences between the stabilized finite element

Keywords and phrases. Nonconforming finite element, Stokes problem, elliptic problem, quadrilateral.

1 Department of Mathematics, Seoul National University, 151-747 Seoul, Korea. ikim@snu.ac.kr; sheen@snu.ac.kr

2 School of Mathematical Sciences, Dalian University of Technology, Dalian, China. zxluo@dlut.edu.cn; mzhl@dlut.edu.cn

3 Interdisciplinary Program in Computational Sciences and Technology, Seoul National University, 151-747 Seoul, Korea.

dongwoosheen@gmail.com; lamyun96@snu.ac.kr

4 Department of Mathematics, Konkuk University, 143-701 Seoul, Korea. cpark@konkuk.ac.kr 
methods and the use of bubble functions in the Galerkin framework . In another direction, the nonconforming finite element methods successfully provide stable numerical solutions. See, for instance, [16,17, 21, 28-30,49] for Stokes and Navier-Stokes problems and $[3,9,27,38,40,43,45,57]$ for elasticity related problems, and the references therein.

In 1973, the nonconforming finite elements for triangles or tetrahedrons were introduced by Crouzeix and Raviart [21]. The idea, at least in the $P_{1}$-nonconforming finite element case, is to employ the DOFs associated with the values at the midpoint of each edge of triangles in $2 \mathrm{D}$ or at the centroid of each face of tetrahedrons in $3 \mathrm{D}$, by replacing those associated with the values at the vertices in the case of the conforming elements. These nonconforming elements were shown to provide stable finite element pairs for the Stokes problem and to give optimal orders of convergence [21], where they together with the piecewise constant element are used to approximate the velocity and pressure fields, respectively.

Even though the triangular or tetrahedral meshes are popular to use, in many cases where the geometry of the problem has a quadrilateral nature, one wishes to use quadrilateral or hexahedral meshes with proper elements. In this direction, nonconforming elements based on quadrilaterals have been proposed by several mathematicians and engineers, including the Wilson element [42,56], which was analyzed by Shi [51]. Han [34] introduced a rectangular element with five local DOFs, Rannacher-Turek [49] presented the rotated $Q_{1}$ nonconforming element of four DOFs, which was modified by Cai-Douglas-Santos-Sheen-Ye $[16,17,25]$ later. Park and Sheen presented the $P_{1}$-nonconforming finite element on quadrilateral meshes which has the lowest DOFs [46]. A posteriori error estimates for simplicial and quadrilateral nonconforming element methods have been developed by Carstensen and $\mathrm{Hu}[18]$. Recently, Altmann and Carstensen introduced the $P_{1}$-nonconforming element for arbitrary triangulations into quadrilaterals and triangles of multiply connected domain [1].

Higher degree nonconforming finite elements have been developed basically by using higher order polynomials on both triangular and quadrilateral meshes. A generalization to higher degree nonconforming elements requires the patch test [37], which implies that a successful $P_{k}$-nonconforming element needs to satisfy a jump condition such that on each interface the jump of polynomials between two adjacent elements should be orthogonal to $P_{k-1}$ polynomials. This implies that a $P_{2}$-nonconforming element, if exists, must be continuous at the two Gauss points on each edge. However, to define the DOFs at the Gauss points causes a trouble due to the existence of a quadratic polynomial which vanishes at the six Gauss points of edges of any triangle and that of a quadratic polynomial which vanishes at the eight Gauss points of edges of any rectangle. Therefore, a special attention is required to be paid when the DOFs for $P_{2}$-nonconforming elements are defined. A successful $P_{2}$-nonconforming element on triangles has been introduced by Fortin and Soulie [30], which is equivalent to an enrichment of the $P_{2}$-conforming element with a nonconforming element-wise bubble function. The three dimensional analogue has been introduced by Fortin [29]. Nonconforming elements based on quadrilaterals have been proposed by Sander and Beckers [50] and analyzed by Shi [52]. Later, Lee and Sheen [41] proposed a $P_{2}$-nonconforming element on rectangles meshes, corresponding to the triangular Fortin-Soulie element. The finite element space proposed in [41] is locally $P_{2} \oplus \operatorname{Span}\left\{x^{2} y, x y^{2}\right\}$ is identical to the incomplete biquadratic element proposed by SanderBeckers [50], but the DOFs are different: the DOFs defined in [50] are the four vertex values and the four edge midpoint values, while those in [41] are the eight values at the two Gauss points on each edge and the integral over the rectangle. However, this element cannot be generalized to the arbitrary quadrilateral meshes. Recently, Köster et al. [39] presented a higher degree nonconforming elements on arbitrary quadrilateral meshes using nonparametric basis functions and additional nonconforming cell bubble functions. Recently, the mimetic finite difference methods have been developed rapidly for general polygonal meshes; for instance, see [11,14,22-24,33], and the references therein. Especially, among the higher-order mimetic finite difference schemes constructed on quadrilateral meshes in [33], the degrees of freedom for a quadratic element consist of one interior value and eight flux normals on edges, which is different from our element to be presented.

The purpose of this paper is to introduce a piecewise $P_{2}$-nonconforming finite element on arbitrary convex quadrilateral meshes that passes the generalized patch test. Our finite element space is locally $P_{2} \oplus \operatorname{Span}\left\{\left(\ell_{13}^{+}\right)^{2},\left(\ell_{24}^{+}\right)^{2}\right\}$ with two ramp functions $\ell_{13}^{+}$, and $\ell_{24}^{+}$defined in (2.3). Indeed, the space has been used as a bivariate spline space on quadrilateral $[44,55]$. Our approach is to use this space as a composite finite 
element to solve second-order elliptic problems and the Stokes problem. For the Stokes problem, we will adopt a proposed piecewise $P_{2}$-nonconforming element for the velocity, and piecewise $P_{1}$-nonconforming element as in [46], for the pressure. We define the DOFs as the eight values at the two Gauss points on each edge, and the value at the intersection of two diagonals of the quadrilateral. Indeed, the DOFs associated with the eight values at the Gauss points are linearly dependent and any seven of them are linearly independent. Thus the total DOFs are eight. Three types of local and global bases are defined. The first and second types of local and global bases are defined associated with vertices and edges. The last type of bases is defined by the value at the intersection of two diagonals. In this case, the basis function vanishes at all Gauss points on the edges, and thus this is essentially a bubble function. After defining local and global interpolations, we derive the optimal order error estimates for second-order elliptic problems and the Stokes problem in broken energy norm. In addition, an optimal order error estimate in $L^{2}(\Omega)$-norm is shown for elliptic problems.

It turns out that our nonconforming finite element space is the union of the conforming piecewise $P_{2}$ and the bubble space, similarly to the $P_{2}$-nonconforming simplicial element of Fortin and Soulie [30].

The contents of the paper are as follows. In Section 2 the piecewise $P_{2}$ spline function space is analyzed and equipped with basis functions. In the following section the piecewise $P_{2}$-nonconforming quadrilateral element is defined. The dimension and basis functions for the Dirichlet and Neumann problems are given. Then in Section 4, projection and interpolation operators are defined and convergence analysis is given. Also, the optimal order error estimates are shown in both discrete energy and $L^{2}$ norms for elliptic problems. In Section 5, the proposed element is applied to solve Stokes equations. An optimal order error estimate in broken energy norm for the Stokes equations is given. Finally, in Section 6, numerical results for the elliptic and Stokes problems are presented.

\section{The PIECEWise $P_{2}$ Spline FunCtion SPACES On QuAdrilaterals}

In this section, we first recall a bivariate spline space on a decomposed quadrilateral $Q[44,55]$, which consists of a piecewise $P_{2}$ polynomial space. We analyze the structure of the space in detail and endow it with suitable DOFs. Local basis functions are constructed. We then define global basis functions.

\subsection{Analysis of the piecewise $P_{2}$ spline function spaces}

For a convex quadrilateral $Q$, denote by $Q^{*}$ the subdivision of $Q$ by connecting its diagonals such that $Q^{*}$ is decomposed into the four non-overlapping triangles $T_{j}, j=1, \cdots, 4$, as shown in Figure 1 . The space of multivariate spline functions $S_{k}^{r}\left(Q^{*}\right)$ is defined by a set of functions which are piecewise polynomials of degree $k$ possessing $r$ th order continuous partial derivatives in $Q$, that is

$$
S_{k}^{r}\left(Q^{*}\right):=\left\{f \in C^{r}(Q)|f|_{T_{j}} \in P_{k}\left(T_{j}\right), j=1, \cdots, 4\right\},
$$

where $P_{k}\left(T_{j}\right)$ denotes the space of polynomials of degree $\leq k$ on $T_{j}$.

Throughout the section, for a convex quadrilateral $Q$, designate by $\mathbf{O}$ the intersection point of two diagonals, by $\mathbf{V}_{j}, j=1, \cdots, 4$, the counterclockwisely numbered vertices of $Q$, by $\mathbf{M}_{j}$ the midpoints of the segments $\overline{\mathbf{V}_{j-1} \mathbf{V}_{j}}$, by $\mathbf{B}_{j}$ and $l_{j}$ the midpoints and lengths of the segments $\overline{\mathbf{O V}_{j}}, j=1, \cdots, 4$, modulo 4, respectively, as shown in Figure 2.

In the case of $S_{2}^{1}\left(Q^{*}\right)$, it is known $[44,55]$ that the dimension of $S_{2}^{1}\left(Q^{*}\right)$ is eight. We will recall this result and show that $f \in S_{2}^{1}\left(Q^{*}\right)$ is uniquely determined by eight values of $f$ at the four vertices and four midpoints of edges of $Q$.

Proposition 2.1. The dimension of $S_{2}^{1}\left(Q^{*}\right)$ is eight. Furthermore, for any given real numbers $a_{j}, a_{j}^{\prime}, j=$ $1, \cdots, 4$, there exists a unique $f \in S_{2}^{1}\left(Q^{*}\right)$ such that

$$
f\left(\mathbf{V}_{j}\right)=a_{j}, f\left(\mathbf{M}_{j}\right)=a_{j}^{\prime}, \quad j=1, \cdots, 4 .
$$




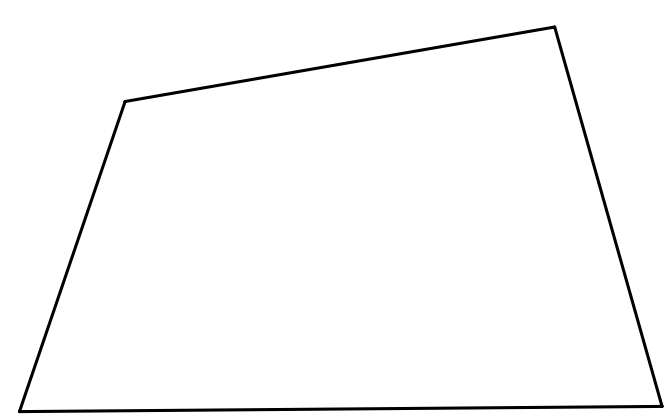

(a) A convex quadrilateral $Q$

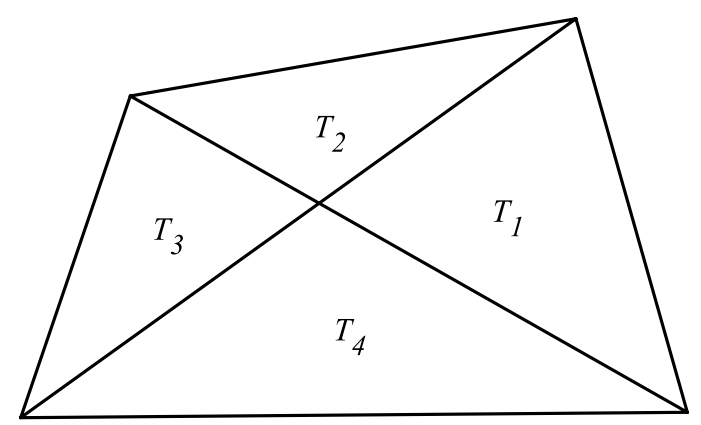

(b) $Q^{*}$ is decomposed into the four non-overlapping triangles $T_{1}, \cdots, T_{4}$

FIgURE 1. A quadrilateral $Q$ and its subdivision $Q^{*}$.

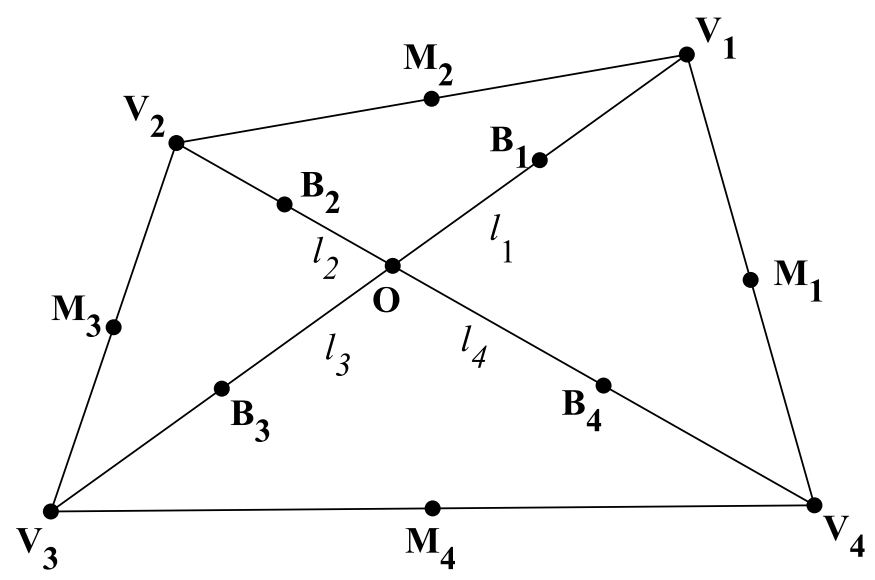

Figure 2. A quadrilateral $Q^{*}$ with vertices $\mathbf{V}_{j}$ 's, edges $\mathbf{E}_{j}$ 's, and midpoints $\mathbf{M}_{j}$ 's. $\mathbf{B}_{j}$ and $l_{j}$ are the midpoints and lengths of the segments $\overline{\mathbf{O V}_{j}}$, respectively.

Proof. Let $\ell_{13}$ and $\ell_{24}$ be the linear polynomials satisfying

$$
\ell_{13}\left(\mathbf{V}_{1}\right)=\ell_{13}\left(\mathbf{V}_{3}\right)=\ell_{24}\left(\mathbf{V}_{2}\right)=\ell_{24}\left(\mathbf{V}_{4}\right)=0 . \quad \ell_{13}\left(\mathbf{V}_{2}\right)=\ell_{24}\left(\mathbf{V}_{1}\right)=1,
$$

Also define the two ramp functions $\ell_{13}^{+}$and $\ell_{24}^{+}$by

$$
\ell_{13}^{+}(x, y)=\max \left(\ell_{13}(x, y), 0\right) \quad \text { and } \quad \ell_{24}^{+}(x, y)=\max \left(\ell_{24}(x, y), 0\right) .
$$

Since $\operatorname{Span}\left\{\left(\ell_{13}^{+}\right)^{2},\left(\ell_{24}^{+}\right)^{2}\right\}$ is a subspace of $S_{2}^{1}\left(Q^{*}\right)$ and its intersection with $P_{2}(Q)$ is null, $S_{2}^{1}\left(Q^{*}\right)$ contains the following linearly independent set

$$
\left\{1, x, y, x y, x^{2}, y^{2},\left(\ell_{13}^{+}\right)^{2},\left(\ell_{24}^{+}\right)^{2}\right\},
$$

which implies that the dimension of $S_{2}^{1}\left(Q^{*}\right)$ is at least eight.

Thus, in order to prove the proposition, it is enough to show that the associated homogeneous system of equations has only the trivial solution. In other words, assume that $f \in S_{2}^{1}\left(Q^{*}\right)$ vanishes at $\mathbf{V}_{j}, \mathbf{M}_{j}, j=1, \cdots, 4$. Then we only have to prove that $f$ is identically zero. 
Let $\xi \in S_{1}^{0}\left(Q^{*}\right)$ be the continuous piecewise linear polynomial such that

$$
\xi(\mathbf{O})=1, \xi\left(\mathbf{V}_{j}\right)=0, \quad j=1, \cdots, 4 .
$$

Since $\xi\left(\mathbf{B}_{j}\right) \neq 0$, for $j=1, \cdots, 4$, there exists a unique continuous piecewise linear polynomial $p \in S_{1}^{0}\left(Q^{*}\right)$ such that

$$
p(\mathbf{O})=f(\mathbf{O}), \quad p\left(\mathbf{B}_{j}\right)=f\left(\mathbf{B}_{j}\right) / \xi\left(\mathbf{B}_{j}\right), \quad j=1, \cdots, 4 .
$$

Since $p \xi$ and $f$ belong to $S_{2}^{0}\left(Q^{*}\right)$ and their values coincide at the thirteen points $\mathbf{O}, \mathbf{V}_{j}, \mathbf{M}_{j}, \mathbf{B}_{j}$, for $j=1, \cdots, 4$, in $Q$, one sees that

$$
f \equiv p \xi
$$

Let $T_{1}=\triangle \mathbf{O V}_{4} \mathbf{V}_{1}$, and $T_{2}=\triangle \mathbf{O V}_{1} \mathbf{V}_{2}$ be two subtriangles in $Q^{*}$ and $p_{j}=\left.p\right|_{T_{j}}, \xi_{j}=\left.\xi\right|_{T_{j}}, j=1,2$. Since $\nabla f \in C^{0}\left(\overline{T_{1} \cup T_{2}}\right), \nabla f$ is well-defined on $\partial T_{1} \cap \partial T_{2}$ and

$$
p\left(\nabla \xi_{2}-\nabla \xi_{1}\right)+\xi\left(\nabla p_{2}-\nabla p_{1}\right)=0 \quad \text { on } \quad \partial T_{1} \cap \partial T_{2} .
$$

Note that $\nabla \xi_{1}$ and $\nabla \xi_{2}$ are constant vectors perpendicular to the segments $\overline{\mathbf{V}_{1} \mathbf{V}_{4}}$ and $\overline{\mathbf{V}_{1} \mathbf{V}_{2}}$, respectively. Hence it follows that $p\left(\mathbf{V}_{1}\right)=0$ due to $\xi\left(\mathbf{V}_{1}\right)=0$ and $\left(\nabla \xi_{2}-\nabla \xi_{1}\right)\left(\mathbf{V}_{1}\right) \neq \mathbf{0}$. A repetition of the argument on the other vertices of $Q$ implies that

$$
p\left(\mathbf{V}_{j}\right)=0, \quad j=1, \cdots, 4 .
$$

Then, $p \equiv f(\mathbf{O}) \xi$, since they belong to $S_{1}^{0}\left(Q^{*}\right)$ and their values coincide at five points $\mathbf{O}, \mathbf{V}_{j}$, for $j=1, \cdots, 4$, in $Q$.

Recalling that $f \equiv p \xi$, we have $f=f(\mathbf{O}) \xi^{2}$. But $\xi^{2} \notin S_{2}^{1}\left(Q^{*}\right)$, since

$$
\nabla \xi_{j}^{2}=2 \xi_{j} \nabla \xi_{j}, \quad \nabla \xi_{j} \neq \nabla \xi_{j+1}
$$

on $\partial T_{j} \cap \partial T_{j+1}$. This shows that $f \equiv 0$ since $f \in S_{2}^{1}\left(Q^{*}\right)$. This completes the proof.

Next, we consider how $f \in S_{2}^{1}\left(Q^{*}\right)$ is determined by the eight values at the vertices and edge midpoints $\mathbf{V}_{j}, \mathbf{M}_{j}, j=1, \cdots, 4$. If we clarify five more values of $f$ at the interior points, $\mathbf{O}, \mathbf{B}_{j}, j=1, \cdots, 4$, then $f$ is uniquely determined on each subtriangle in $Q^{*}$, separately. With the aid of following Lemma, we will address an explicit form of $f \in S_{2}^{1}\left(Q^{*}\right)$, which is useful, in the subsequent Theorem 2.3.

Lemma 2.2. Let a triangle $\triangle A B C$ be divided into two triangles $T_{1}=\triangle O C B$ and $T_{2}=\triangle O C A$ by a point $O$ on the segment $\overline{A B}$ as shown in Figure 3. Denote the midpoints of $\overline{O A}, \overline{O B}, \overline{C A}, \overline{C B}$ and $\overline{C O}$ by $O_{A}, O_{B}, C_{A}, C_{B}$ and $C_{O}$, respectively. Let $g \in C^{0}\left(T_{1} \cup T_{2}\right)$ satisfy $\left.g\right|_{T_{j}} \in P_{2}\left(T_{j}\right), j=1,2$. Then the following condition is sufficient and necessary for $g \in C^{1}\left(T_{1} \cup T_{2}\right)$.

$$
D_{g}\left(C_{A}, C_{O}\right)-D_{g}\left(C_{O}, C_{B}\right)=D_{g}\left(O_{A}, O\right)-D_{g}\left(O, O_{B}\right)=\frac{1}{2}\left(D_{g}(A, O)-D_{g}(O, B)\right),
$$

where

$$
D_{g}(Q, R)=\frac{g(Q)-g(R)}{|Q-R|}, \quad Q, R \in \mathbb{R}^{2}
$$

Proof. Let $g \in C^{0}\left(T_{1} \cup T_{2}\right)$ satisfy $\left.g\right|_{T_{j}} \in P_{2}\left(T_{j}\right), j=1$, 2. Set $g_{1}=\left.g\right|_{T_{1}}$ and $g_{2}=\left.g\right|_{T_{2}}$ and let $\eta$ be the unit vector parallel to the vector $\overrightarrow{A B}$. Notice that $g \in C^{1}\left(T_{1} \cup T_{2}\right)$ if and only if

$$
\frac{\partial g_{1}}{\partial \eta}(O)=\frac{\partial g_{2}}{\partial \eta}(O), \quad \frac{\partial g_{1}}{\partial \eta}(C)=\frac{\partial g_{2}}{\partial \eta}(C)
$$

since the directional derivatives $\frac{\partial g_{1}}{\partial \eta}$ and $\frac{\partial g_{2}}{\partial \eta}$ are linear functions. 


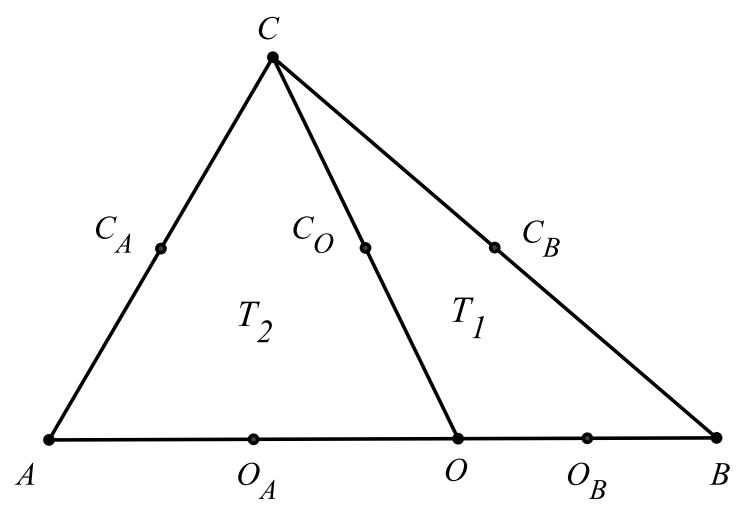

Figure 3. Points and subtriangles in a triangle $\triangle A B C$.

It is useful to notice that a univariate function $p$ on the interval $[a, b]$ satisfies

$$
p^{\prime}\left(\frac{a+b}{2}\right)=\frac{p(b)-p(a)}{b-a}
$$

whenever $p$ is quadratic. We utilize (2.6) to have

$$
\begin{aligned}
\frac{\partial g_{1}}{\partial \eta}\left(\frac{C_{A}+C_{O}}{2}\right) & =-D_{g_{1}}\left(C_{A}, C_{O}\right), \\
\frac{\partial g_{1}}{\partial \eta}\left(\frac{O_{A}+O}{2}\right) & =-D_{g_{1}}\left(O_{A}, O\right), \\
\frac{\partial g_{1}}{\partial \eta}\left(O_{A}\right) & =-D_{g_{1}}(A, O) .
\end{aligned}
$$

Since $\frac{\partial g_{1}}{\partial \eta}$ is linear and $\frac{C_{A}+C_{O}}{2}$ and $\frac{O_{A}+O}{2}$ are the midpoints of the segments $\overline{C O_{A}}$ and $\overline{O_{A} O}$, respectively, we have, from (2.7),

$$
\begin{gathered}
\frac{\partial g_{1}}{\partial \eta}(C)=2 \frac{\partial g_{1}}{\partial \eta}\left(\frac{C_{A}+C_{O}}{2}\right)-\frac{\partial g_{1}}{\partial \eta}\left(O_{A}\right)=-2 D_{g}\left(C_{A}, C_{O}\right)+D_{g}(A, O) \\
\frac{\partial g_{1}}{\partial \eta}(O)=2 \frac{\partial g_{1}}{\partial \eta}\left(\frac{O_{A}+O}{2}\right)-\frac{\partial g_{1}}{\partial \eta}\left(O_{A}\right)=-2 D_{g}\left(O_{A}, O\right)+D_{g}(A, O)
\end{gathered}
$$

By the same argument for $g_{2}$, we establish

$$
\begin{aligned}
& \frac{\partial g_{2}}{\partial \eta}(C)=-2 D_{g}\left(C_{O}, C_{B}\right)+D_{g}(O, B), \\
& \frac{\partial g_{2}}{\partial \eta}(O)=-2 D_{g}\left(O, O_{B}\right)+D_{g}(O, B) .
\end{aligned}
$$

From (2.8) and (2.9), the conditions in (2.5) are equivalent to (2.4), which completes the proof.

Theorem 2.3. Let $f \in S_{2}^{0}\left(Q^{*}\right)$. Then $f \in S_{2}^{1}\left(Q^{*}\right)$ if and only if the values of $f$ at the eight boundary points $\mathbf{V}_{j}, \mathbf{M}_{j}, j=1, \cdots, 4$, and the five interior points $\mathbf{O}, \mathbf{B}_{j}, j=1, \cdots, 4$, satisfy the following relationships:

$$
f(\mathbf{O})=\frac{2}{\left(l_{1}+l_{3}\right)\left(l_{2}+l_{4}\right)} \sum_{j=1}^{4} f\left(\mathbf{M}_{j}\right) l_{j+1} l_{j+2}-\frac{1}{2}\left(\frac{l_{1} f\left(\mathbf{V}_{3}\right)+l_{3} f\left(\mathbf{V}_{1}\right)}{l_{1}+l_{3}}+\frac{l_{2} f\left(\mathbf{V}_{4}\right)+l_{4} f\left(\mathbf{V}_{2}\right)}{l_{2}+l_{4}}\right)
$$




$$
f\left(\mathbf{B}_{j}\right)=\frac{l_{j+1} f\left(\mathbf{M}_{j}\right)+l_{j-1} f\left(\mathbf{M}_{j+1}\right)}{l_{j+1}+l_{j-1}}-\frac{1}{4}\left(\frac{l_{j+1} f\left(\mathbf{V}_{j-1}\right)+l_{j-1} f\left(\mathbf{V}_{j+1}\right)}{l_{j+1}+l_{j-1}}-f(\mathbf{O})\right), \quad j=1, \cdots, 4,
$$

where the indices are calculated up to modulo 4.

The equation (2.10) implies that the value of a function $f \in S_{2}^{0}\left(Q^{*}\right)$ at the intersection of diagonals is uniquely determined by the function values at $\mathbf{V}_{j}, \mathbf{M}_{j}, j=1, \cdots, 4$. Equation (2.11) means that the values at the midpoints between the intersection of diagonals and the vertex points are determined by those at $\mathbf{O}, \mathbf{V}_{j}$ and $\mathbf{M}_{j}, j=1, \cdots, 4$.

Proof. Let $f \in S_{2}^{1}\left(Q^{*}\right)$. Then (2.11) follows from Lemma 2.2 considering the triangle $\triangle V_{j-1} V_{j} V_{j+1}$, we have

$$
\frac{2}{l_{j-1}}\left(f\left(\mathbf{M}_{j}\right)-f\left(\mathbf{B}_{j}\right)\right)-\frac{2}{l_{j+1}}\left(f\left(\mathbf{B}_{j}\right)-f\left(\mathbf{M}_{j+1}\right)\right)=\frac{1}{2 l_{j-1}}\left(f\left(\mathbf{V}_{j-1}\right)-f(\mathbf{O})\right)-\frac{1}{2 l_{j+1}}\left(f(\mathbf{O})-f\left(\mathbf{V}_{j+1}\right)\right),
$$

which implies (2.11) for $f\left(\mathbf{B}_{j}\right)$. In order to prove (2.10), we apply Lemma 2.2 again to the triangle $\triangle V_{j-1} V_{j} V_{j+1}$ to get

$$
\frac{2}{l_{j-1}}\left(f\left(\mathbf{B}_{j-1}\right)-f(\mathbf{O})\right)-\frac{2}{l_{j+1}}\left(f(\mathbf{O})-f\left(\mathbf{B}_{j+1}\right)\right)=\frac{1}{2 l_{j-1}}\left(f\left(\mathbf{V}_{j-1}\right)-f(\mathbf{O})\right)-\frac{1}{2 l_{j+1}}\left(f(\mathbf{O})-f\left(\mathbf{V}_{j+1}\right)\right) .
$$

Eliminating $f\left(\mathbf{B}_{j-1}\right)$ and $f\left(\mathbf{B}_{j+1}\right)$ from (2.12) with the aid of (2.11), we establish (2.10).

Conversely, let $f \in S_{2}^{0}\left(Q^{*}\right)$ satisfy (2.10) and (2.11). Then we will show $f \in S_{2}^{1}\left(Q^{*}\right)$. By Proposition 2.1, there exists $g \in S_{2}^{1}\left(Q^{*}\right)$ such that

$$
g\left(\mathbf{V}_{j}\right)=f\left(\mathbf{V}_{j}\right) \text { and } \quad g\left(\mathbf{M}_{j}\right)=f\left(\mathbf{M}_{j}\right), \quad j=1, \cdots, 4 .
$$

Since $g \in S_{2}^{1}\left(Q^{*}\right)$ should satisfy $(2.10),(2.11)$, we have

$$
g(\mathbf{O})=f(\mathbf{O}), \quad g\left(\mathbf{B}_{j}\right)=f\left(\mathbf{B}_{j}\right), \quad j=1, \cdots, 4 .
$$

Then, in each subtriangle in $Q^{*}, f$ and $g$ are quadratic and agree with the values at vertices and midpoints. This of course means $f \equiv g$, and thus $f \in S_{2}^{1}\left(Q^{*}\right)$.

We now investigate the relation of $f \in S_{2}^{1}\left(Q^{*}\right)$ on the values at the Gauss points of each edge in $Q$. Let $\mathbf{G}_{2 j-1}$ and $\mathbf{G}_{2 j}$ be the Gauss points on the segments $\overline{\mathbf{V}_{j-1} \mathbf{V}_{j}}, j=1, \cdots, 4$, where the indices are counterclockwisely numbered as depicted in Figure 4.

The following properties of univariate quadratic functions are useful: let $p$ be a quadratic function and $g_{j}, j=1,2$, the two Gauss points for the interval $[a, b]$ such that $g_{1}<g_{2}$, then $p$ satisfies that

$$
\begin{gathered}
p\left(g_{1}\right)+p\left(g_{2}\right)=\frac{4}{3} p\left(\frac{a+b}{2}\right)+\frac{1}{3}(p(a)+p(b)), \\
p\left(g_{1}\right)-p\left(g_{2}\right)=\frac{1}{\sqrt{3}}(p(a)-p(b)) .
\end{gathered}
$$

We then have the following proposition.

Proposition 2.4. For any $f \in S_{2}^{0}\left(Q^{*}\right)$, the following relationship holds:

$$
\sum_{j=1}^{4}\left(f\left(\mathbf{G}_{2 j}\right)-f\left(\mathbf{G}_{2 j-1}\right)\right)=0 .
$$




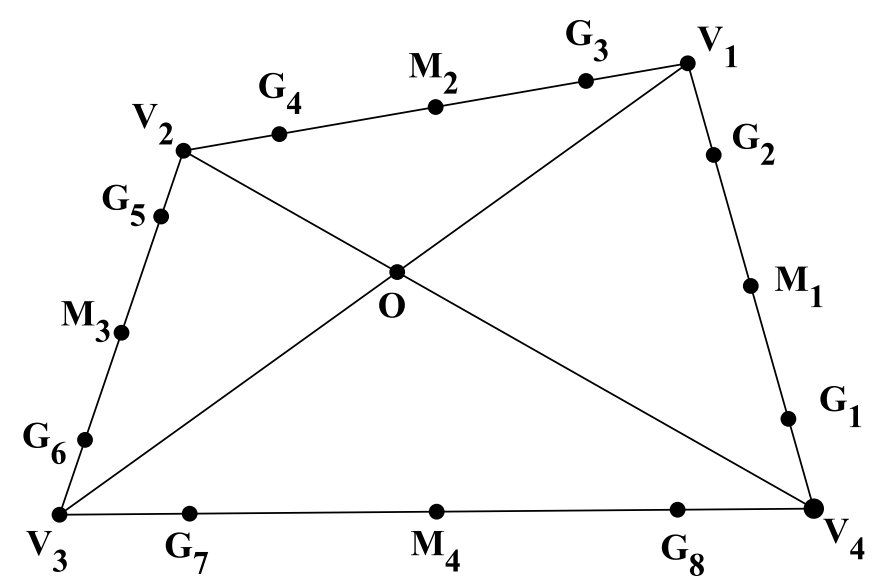

Figure 4. Gauss points $\mathbf{G}_{j}, j=1, \cdots, 8$ in $Q^{*}$.

Proof. Since $f \in S_{2}^{0}\left(Q^{*}\right)$ is quadratic on each edge of $Q$, it follows from (2.13) that

$$
f\left(\mathbf{G}_{2 j}\right)-f\left(\mathbf{G}_{2 j-1}\right)=\frac{1}{\sqrt{3}}\left(f\left(\mathbf{V}_{j}\right)-f\left(\mathbf{V}_{j-1}\right)\right), \quad j=1, \cdots, 4 .
$$

Therefore $\sum_{j=1}^{4}\left(f\left(\mathbf{G}_{2 j}\right)-f\left(\mathbf{G}_{2 j-1}\right)\right)=0$ due to the convention $\mathbf{V}_{4}=\mathbf{V}_{0}$.

The following proposition is immediate, but useful, which gives explicit formula at the Gauss points if the values at the vertices and midpoints are given.

Proposition 2.5. Let $p \in S_{2}^{k}\left(Q^{*}\right), k=0,1$ be given. Then the values at the Gauss points are given by

$$
\begin{aligned}
p\left(\mathbf{G}_{2 j-1}\right) & =\frac{2}{3} p\left(\mathbf{M}_{j}\right)+\frac{p\left(\mathbf{V}_{j-1}\right)+p\left(\mathbf{V}_{j}\right)}{6}+\frac{1}{2 \sqrt{3}}\left[p\left(\mathbf{V}_{j-1}\right)-p\left(\mathbf{V}_{j}\right)\right], \\
p\left(\mathbf{G}_{2 j}\right) & =\frac{2}{3} p\left(\mathbf{M}_{j}\right)+\frac{p\left(\mathbf{V}_{j-1}\right)+p\left(\mathbf{V}_{j}\right)}{6}-\frac{1}{2 \sqrt{3}}\left[p\left(\mathbf{V}_{j-1}\right)-p\left(\mathbf{V}_{j}\right)\right],
\end{aligned}
$$

for $j=1, \cdots, 4$. Moreover, if $k=1$, then (2.10) should be fulfilled.

Proof. The proof is an easy consequence of (2.13) and Theorem 2.3.

Finally, the following theorem is a converse to the above Proposition 2.5 which describes the formula at the vertices and midpoints given the values at the Gauss points.

Theorem 2.6. For any given real numbers $a_{j}, j=0, \cdots, 8$, satisfying

$$
\sum_{j=1}^{4}\left(a_{2 j}-a_{2 j-1}\right)=0,
$$

there exists a unique $f \in S_{2}^{1}\left(Q^{*}\right)$ such that $f\left(\mathbf{G}_{j}\right)=a_{j}$, for $j=1, \cdots, 8$, and $f(\mathbf{O})=a_{0}$.

Furthermore, let $b_{j}=a_{2 j}-a_{2 j-1}$, for $j=1, \cdots, 4$, then $f \in S_{2}^{1}\left(Q^{*}\right)$ is uniquely determined by setting

$$
\begin{aligned}
& f\left(\mathbf{V}_{j}\right)=\alpha+\frac{\sqrt{3}}{4}\left(b_{j-1}+2 b_{j}-b_{j+1}\right), \quad j=1, \cdots, 4, \\
& f\left(\mathbf{M}_{j}\right)=-\frac{\alpha}{2}+\frac{3}{4}\left(a_{2 j}+a_{2 j-1}\right)-\frac{\sqrt{3}}{8}\left(b_{j-1}-b_{j+1}\right), \quad j=1, \cdots, 4,
\end{aligned}
$$

where the indices of $b$ are modulo 4 and $\alpha$ is calculated from (2.10). 
Proof. Suppose $a_{j}, j=0, \cdots, 8$, are given fulfilling (2.17) and set $b_{j}=a_{2 j}-a_{2 j-1}$, for $j=1, \cdots, 4$. Plugging into (2.18) the formulae for $f\left(\mathbf{V}_{j}\right)$ and $f\left(\mathbf{M}_{j}\right)$ in $(2.10)$ and $f(\mathbf{O})=a_{0}$, one obtains a linear equation in $\alpha$. Indeed, the coefficient of $\alpha$ is given by

$$
-2 \frac{\sum_{j=1}^{4} l_{j+1} l_{j+2}}{\left(l_{1}+l_{3}\right)\left(l_{2}+l_{4}\right)}=-2,
$$

which is nonzero. Therefore, for given $\alpha \in \mathbb{R}$, by Theorem 2.3, there exists $f \in S_{2}^{1}\left(Q^{*}\right)$ which fulfills $(2.18)$. Moreover, such an $f \in S_{2}^{1}\left(Q^{*}\right)$ satisfies $f(\mathbf{O})=a_{0}$ and we can easily verify $f\left(\mathbf{G}_{j}\right)=a_{j}, j=1, \cdots, 8$, using the values of $f$ in (2.13) and (2.18).

To prove uniqueness, suppose that $f \in S_{2}^{1}\left(Q^{*}\right)$ satisfy

$$
f(\mathbf{O})=0, \quad f\left(\mathbf{G}_{j}\right)=0, j=1, \cdots, 8 .
$$

If $f\left(\mathbf{V}_{1}\right)=c$, then, from $(2.13)$, we get

$$
f\left(\mathbf{V}_{j}\right)=c, \quad f\left(\mathbf{M}_{j}\right)=-\frac{c}{2}, \quad j=1, \cdots, 4 .
$$

Applying Theorem 2.3, we have

$$
f(\mathbf{O})=\left(\frac{-\sum_{j=1}^{4} l_{j+1} l_{j+2}}{\left(l_{1}+l_{3}\right)\left(l_{2}+l_{4}\right)}-1\right) c=-2 c .
$$

From $f(\mathbf{O})=0$ it follows that $c=0$. Thus $f$ vanishes and this completes our proof.

Remark 2.7. Theorem 2.6 guarantees the existence of four vertex-based functions whose values are 1 at the two nearest Gauss points from each given vertex and 0 at the other six Gauss points and at the intersection point of diagonals. Similarly, we have four edge-based functions whose values are 1 at the two Gauss points for each given edge and 0 at the other six Gauss points and at the intersection point of diagonals. Lastly, there is one bubble-type function whose values are 1 at the intersection point of diagonals and 0 at all the Gauss points.

\subsection{Basis functions for the piecewise $P_{2}$ spline function space}

So far, we have analyzed the structure of a piecewise $P_{2}$ spline function space $S_{2}^{1}\left(Q^{*}\right)$, and supplied some suggestions for endowing it with suitable DOFs in Remark 2.7. Thus we proceed to define the eight local basis functions by using any seven values at the eight Gauss points plus one bubble function based on Theorem 2.6.

Define the four vertex-wise local basis functions (see Fig. 5a) by

$$
\phi^{\mathbf{V}_{j}}\left(\mathbf{G}_{k}\right)=\left\{\begin{array}{l}
1, k=2 j, 2 j+1 \\
0, \text { otherwise }
\end{array} \text { and } \quad \phi^{\mathbf{V}_{j}}(\mathbf{O})=0, \quad j=1, \cdots, 4,\right.
$$

the four edge-wise local basis functions (see Fig. 5b) by

$$
\phi^{\mathbf{E}_{j}}\left(\mathbf{G}_{k}\right)=\left\{\begin{array}{l}
1, k=2 j-1,2 j \\
0, \text { otherwise }
\end{array} \text { and } \quad \phi^{\mathbf{E}_{j}}(\mathbf{O})=0, \quad j=1, \cdots, 4,\right.
$$

and the bubble function (see Fig. 5c) by

$$
\phi^{Q}(\mathbf{O})=1 \text { and } \quad \phi^{Q}\left(\mathbf{G}_{j}\right)=0, \quad j=1, \cdots, 8 .
$$




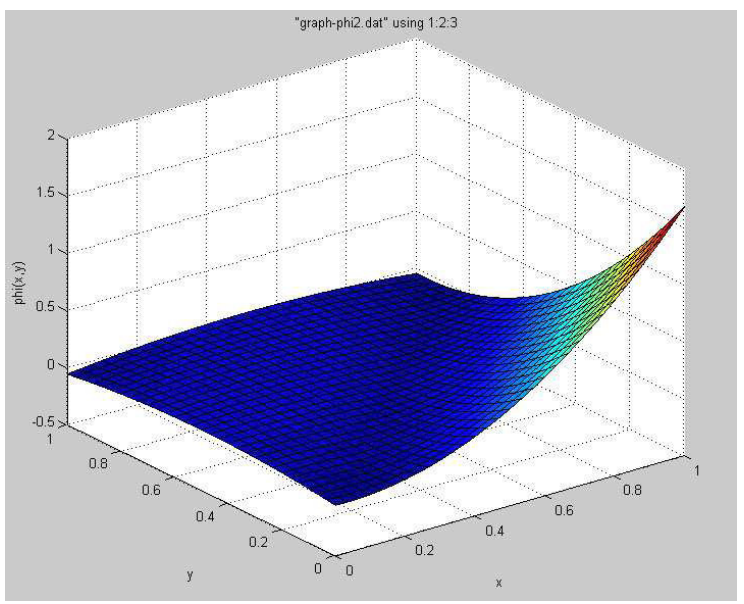

(a) The vertex-wise nonconforming local basis function $\phi^{\mathbf{V}}(\mathbf{x})$.

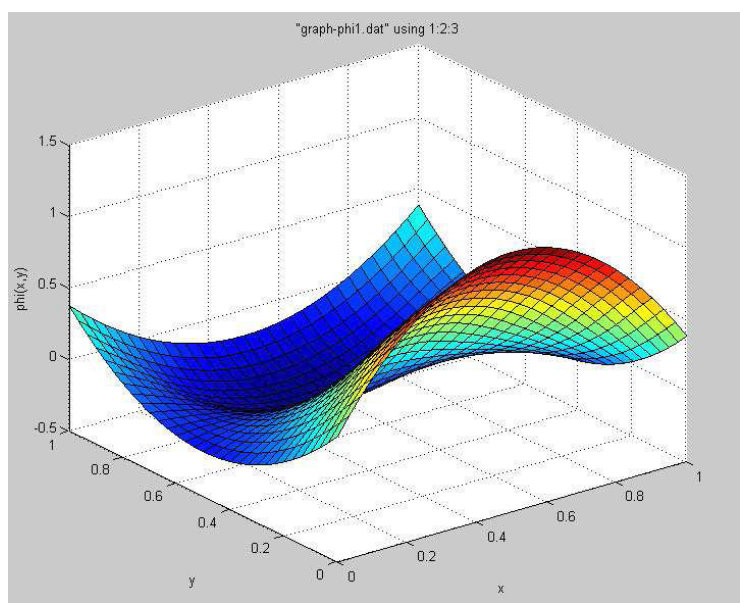

(b) The edge-wise nonconforming local basis function $\phi^{\mathbf{E}}(\mathbf{x})$.

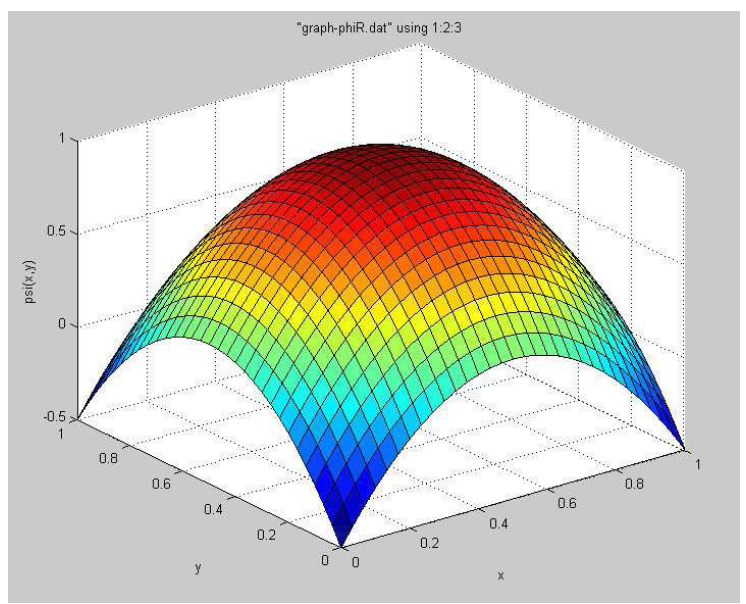

(c) The bubble type nonconforming local basis function $\phi^{Q}(\mathbf{x})$.

Figure 5. Shapes of local basis functions $\phi^{\mathbf{V}}(\mathbf{x})$ in (2.19), $\phi^{\mathbf{E}}(\mathbf{x})$ in $(2.20)$ and $\phi^{Q}(\mathbf{x})$ in $(2.21)$.

\section{The PIECEWISE $P_{2}$-NONCONFORMING ELEMENT ON QUADRILATERALS}

Based on the analysis of the previous section we are ready to define a piecewise $P_{2}$-nonconforming quadrilateral element.

Define the piecewise $P_{2}$-nonconforming quadrilateral element $\left(Q, P_{Q}, \Sigma_{Q}\right)$ as follows:

- $Q$ is a convex quadrilateral;

- The (piecewise) polynomial space is given by $P_{Q}=S_{2}^{1}\left(Q^{*}\right)$;

- The degrees of freedom are given by $\Sigma_{Q}=\left\{\phi\left(\mathbf{G}_{j}\right), j=1, \cdots, 7 ; \phi(\mathbf{O})\right\}$ for every $\phi \in P_{Q}$.

Alternatively, due to Proposition 2.5 and Theorem 2.6, the degrees of freedom may be defined as follows:

$$
\Sigma_{Q}^{\prime}=\left\{\phi\left(\mathbf{V}_{j}\right), \phi\left(\mathbf{M}_{j}\right), j=1, \cdots, 4 ; \phi(\mathbf{O})\right\} \text { for every } \phi \in P_{Q} .
$$




\subsection{The piecewise $P_{2}$-nonconforming finite element space}

Let $\Omega$ be a simply connected polygonal domain with the Lipschitz-continuous boundary $\partial \Omega$ and $\mathcal{T}_{h}=\cup_{j=1}^{N_{Q}} Q_{j}$ be a triangulation of the domain $\Omega$ by non-overlapping convex quadrilaterals $Q_{j}$ 's such that $\bar{\Omega}=\cup_{j=1}^{N_{Q}} \overline{Q_{j}}$ with $\operatorname{diam}\left(Q_{j}\right) \leq h$, where $N_{Q}$ is the number of quadrilaterals. Let $Q_{j}^{*}$ be the union of subdivision $T_{j}$ by connecting its diagonals. Then set $\mathcal{T}_{h}^{*}=\cup_{j=1}^{N_{Q}} Q_{j}^{*}$.

Let $N_{V}, N_{E}$ and $N_{G}$ denote the number of vertices, edges and Gauss points, respectively, in $\mathcal{T}_{h}$. Set

$$
\begin{aligned}
\mathcal{V}_{h} & =\left\{\mathbf{V}_{1}, \mathbf{V}_{2}, \cdots, \mathbf{V}_{N_{V}}\right\}: \text { the set of all vertices in } \mathcal{T}_{h}, \\
\mathcal{E}_{h} & =\left\{\mathbf{E}_{1}, \mathbf{E}_{2}, \cdots, \mathbf{E}_{N_{E}}\right\}: \text { the set of all edges in } \mathcal{T}_{h}, \\
\mathcal{G}_{h} & =\left\{\mathbf{G}_{1}, \mathbf{G}_{2}, \cdots, \mathbf{G}_{N_{G}}\right\}: \text { the set of all Gauss points on the edges in } \mathcal{T}_{h}, \\
\mathcal{M}_{h} & =\left\{\mathbf{M}_{1}, \mathbf{M}_{2}, \cdots, \mathbf{M}_{N_{E}}\right\}: \text { the set of all midpoints on the edges in } \mathcal{T}_{h}, \\
\mathcal{O}_{h} & =\left\{\mathbf{O}_{1}, \mathbf{O}_{2}, \cdots, \mathbf{O}_{N_{Q}}\right\}: \text { the set of all intersections of the two diagonals of quadrilaterals in } \mathcal{T}_{h} .
\end{aligned}
$$

In particular, let $N_{V}^{i}, N_{E}^{i}$ and $N_{G}^{i}$ denote the number of interior vertices, edges, and Gauss points, respectively. Also, $\mathcal{E}_{h}^{i}$ and $\mathcal{E}_{h}^{b}$ will designate the sets of all interior and boundary edges, respectively.

Our objective is to introduce a piecewise $P_{2}$-nonconforming finite element space associated with the quadrilateral decomposition $\mathcal{T}_{h}$. Set

$$
\begin{aligned}
\mathcal{N} C_{2}^{h}= & \left\{v_{h}: \Omega \rightarrow \mathbb{R}\left|v_{h}\right|_{Q} \in S_{2}^{1}\left(Q^{*}\right) \text { for all } Q \in \mathcal{T}_{h},\right. \\
& \left.v_{h} \text { is continuous at every Gauss point } \mathbf{G} \in \mathcal{G}_{h} \text { that is not on } \partial \Omega\right\}, \\
\mathcal{N} C_{2,0}^{h}= & \left\{v_{h} \in \mathcal{N} C_{2}^{h} \mid v_{h} \text { vanishes at every Gauss point on } \partial \Omega\right\} .
\end{aligned}
$$

We also consider the piecewise $P_{2}$-conforming spaces:

$$
\begin{aligned}
X_{h} & =\left\{v_{h} \in C^{0}(\Omega)\left|v_{h}\right|_{Q} \in S_{2}^{1}\left(Q^{*}\right) \quad \forall Q \in \mathcal{T}_{h}\right\}, \\
X_{0, h} & =\left\{v_{h} \in X_{h} \mid v_{h} \text { vanishes on } \partial \Omega\right\},
\end{aligned}
$$

with the bubble space $\Phi_{h}$ given as follows:

$$
\Phi_{h}=\left\{\phi_{h} \in \mathcal{N} C_{2}^{h} \mid \phi_{h}(\mathbf{G})=0 \text { for all } \mathbf{G} \in \mathcal{G}_{h}\right\} .
$$

\subsection{Global basis functions for $\mathcal{N} C_{2}^{h}$ and $\mathcal{N} C_{2,0}^{h}$ and their dimensions}

For each vertex $\mathbf{V}_{j} \in \mathcal{V}_{h}$, denote by $\mathcal{E}_{h}(j)$ and $\mathcal{G}_{h}(j)$ the set of all edges $\mathbf{E} \in \mathcal{E}_{h}$ with one of the endpoints being $\mathbf{V}_{j}$ and the set of Gauss points nearer to $\mathbf{V}_{j}$ of the two Gauss points on $\mathbf{E}$ for all $\mathbf{E} \in \mathcal{E}_{h}(j)$, respectively. Utilizing the three types of local basis functions given in (2.19), (2.20), and (2.21), we define the three types of global basis functions for $\mathcal{N C}_{2}^{h}$.

Definition 3.1. The first type of global basis functions are associated with vertices $\mathbf{V}_{j} \in \mathcal{V}_{h}$ (see Fig. 6a). Define $\varphi_{j}^{V} \in \mathcal{N} C_{2}^{h}, j=1, \cdots, N_{V}$, by

$$
\begin{aligned}
\varphi_{j}^{V}(\mathbf{G}) & = \begin{cases}1, & \mathbf{G} \in \mathcal{G}_{h}(j), \\
0, & \text { otherwise, }\end{cases} \\
\varphi_{j}^{V}\left(\mathbf{O}_{k}\right) & =0 \text { for all } k=1, \cdots, N_{Q} .
\end{aligned}
$$

The second type of global basis functions are associated with edges $\mathbf{E}_{j} \in \mathcal{E}_{h}$ (see Fig. 6b). Define $\varphi_{j}^{E} \in \mathcal{N} C_{2}^{h}, j=$ $1, \cdots, N_{E}$, by

$$
\begin{aligned}
& \varphi_{j}^{E}(\mathbf{G})= \begin{cases}1, & \mathbf{G} \text { is a Gauss point on } \mathbf{E}_{j}, \\
0, & \text { otherwise, }\end{cases} \\
& \varphi_{j}^{E}\left(\mathbf{O}_{k}\right)=0 \text { for all } k=1, \cdots, N_{Q} .
\end{aligned}
$$




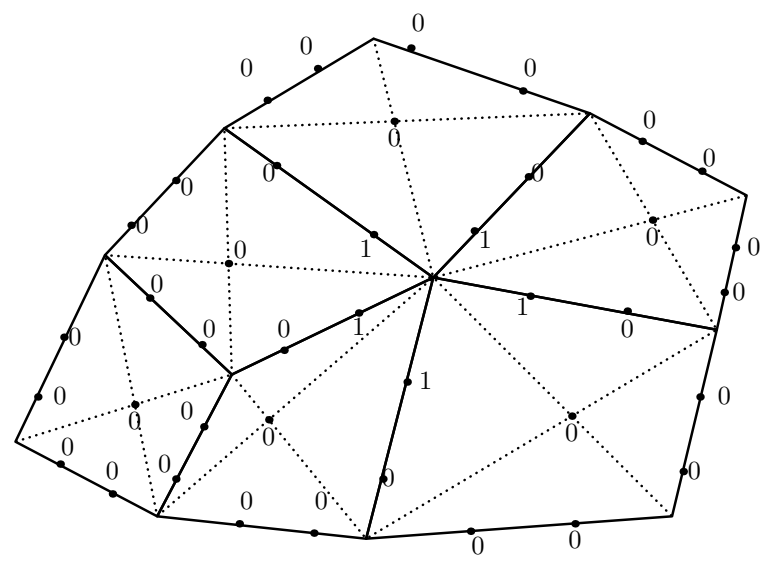

(a) The first type global basis function is associated with a vertex.

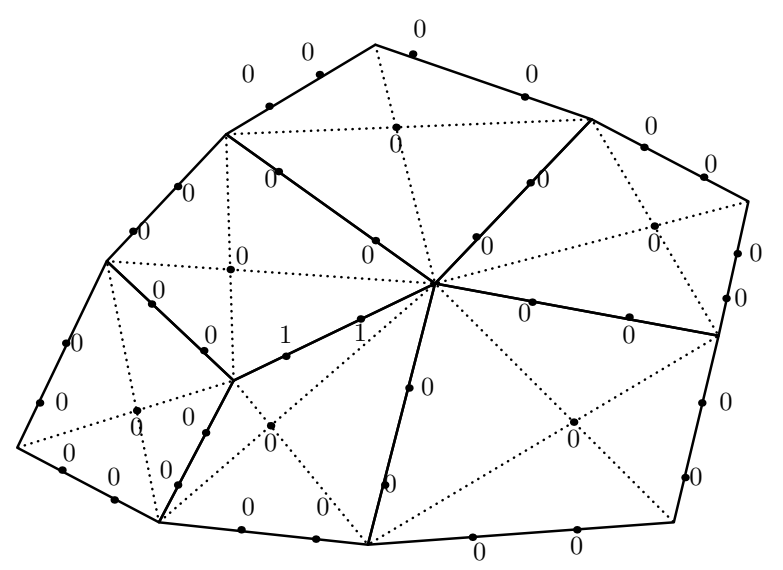

(b) The second type global basis function is associated with an edge.

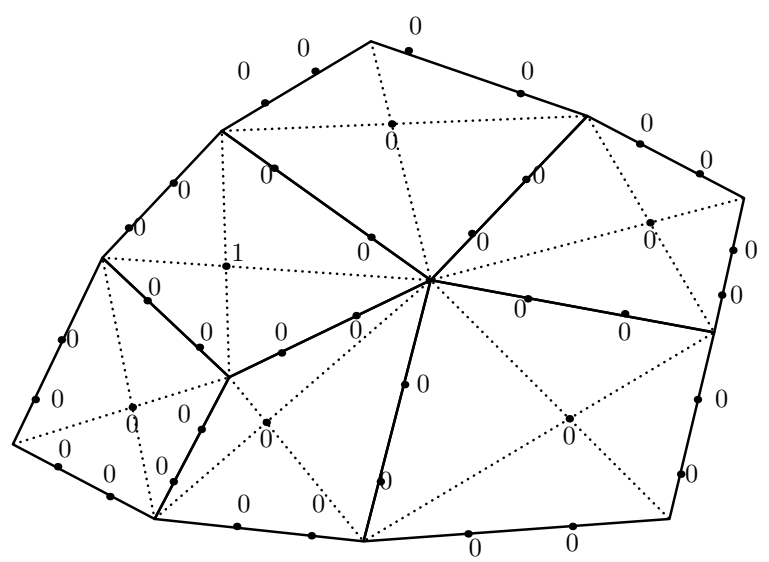

(c) The third type global basis function is associated with a quadrilateral.

Figure 6 . The global basis functions.

The last type of global basis functions are associated with the quadrilaterals (see Fig. 6c). Define $\varphi_{j}^{Q} \in \mathcal{N} C_{2}^{h}, j=$ $1, \cdots, N_{Q}$, by

$$
\varphi_{j}^{Q}\left(\mathbf{G}_{k}\right)=0, \text { for all } k=1, \cdots, N_{G}, \quad \text { and } \quad \varphi_{j}^{Q}\left(\mathbf{O}_{k}\right)=\delta_{j k}, \text { for all } k=1, \cdots, N_{Q} .
$$

Similarly, define the three types of functions which will serve as global basis functions for $\mathcal{N} C_{2,0}^{h}$ with those for $\mathcal{N} C_{2}^{h}$ excluding $\phi_{j}^{V}$ 's which are associated with boundary vertices and $\phi_{j}^{E}$ 's which are are associated with boundary edges.

Now let us present the dimensions for the nonconforming finite element spaces $\mathcal{N} C_{2}^{h}$ and $\mathcal{N} C_{2,0}^{h}$. We begin by invoking that $X_{h}$ is a conforming finite element space whose local DOFs consist of the values at the four vertices and four midpoints on each quadrilateral by Proposition 2.1. We have the following observation which is similar to the quadratic nonconforming element on triangles of Fortin and Soulie [30].

Theorem 3.2. Any function in $\mathcal{N} C_{2}^{h}$ can be written as the sum of a function in piecewise $S_{2}^{1}$-conforming finite element space $X_{h}$ and a function in bubble space $\Phi_{h}$. This representation can be made uniquely by specifying 
one function value at any vertex in bubble space, that is $\mathcal{N} C_{2}^{h}=X_{h}+\Phi_{h}$ and $\operatorname{dim}\left(X_{h} \cap \Phi_{h}\right)=1$. Moreover, $\operatorname{dim}\left(\mathcal{N} C_{2}^{h}\right)=2 N_{E}=N_{E}+N_{V}+N_{Q}-1$.

Proof. It is clear that $X_{h}+\Phi_{h} \subset \mathcal{N} C_{2}^{h}$ since adding to $v_{h} \in X_{h}$ a bubble function $\phi^{Q}$ on each $Q$ preserves the continuity at the Gauss points. To prove equality we consider the dimensions of $X_{h}+\Phi_{h}$ and $\mathcal{N} C_{2}^{h}$. First, we prove that the intersection of $X_{h}$ and $\Phi_{h}$ is one-dimensional. Let us choose the function $\phi_{h} \in X_{h} \cap \Phi_{h}$ with $\phi_{h}\left(\mathbf{V}_{j}\right)=\alpha$ for some $j$ and $\phi_{h}\left(\mathbf{G}_{k}\right)=0$ for all $k$. Since $\phi_{h}$ has the same value (zero value) at all Gauss points and $\phi_{h} \in S_{2}^{1}\left(Q^{*}\right)$, one sees that $\phi_{h}\left(\mathbf{V}_{k}\right)=\alpha$ for all $k$ due to (2.13). This gives the whole information on each edge including the midpoint values due to Theorem 2.3. Therefore, with the eight function values at four vertices and four midpoints, $\phi_{h} \in X_{h} \cap \Phi_{h}$ is uniquely determined by the constant value at $\mathbf{V}_{j}$. By [41] and $X_{h}+\Phi_{h} \subset \mathcal{N} C_{2}^{h}$, we have

$$
\operatorname{dim} X_{h}+\operatorname{dim} \Phi_{h}-1=\operatorname{dim}\left(X_{h}+\Phi_{h}\right) \leq \operatorname{dim} \mathcal{N} C_{2}^{h} \leq 2 N_{E} .
$$

For $X_{h}$ there are $N_{E}+N_{V}$ DOFs and for $\Phi_{h}$ there are $N_{Q}$ DOFs. Thus, the number of DOFs of $X_{h}+\Phi_{h}$ is $\left(N_{E}+N_{V}\right)+N_{Q}-1=N_{E}+\left(N_{V}+N_{Q}-1\right)=2 N_{E}$. This completes the proof.

Remark 3.3. From Theorem 3.2 , it follows that $\mathcal{N} C_{2}^{h}$ is nothing but a standard piecewise $P_{2}$-conforming finite element space enriched by the space of bubble functions $\Phi_{h}=\operatorname{Span}\left\{\phi_{j}^{Q}\right.$ : for $\left.j=1, \cdots, N_{Q}\right\}$.

Now, we construct global basis functions for the piecewise $P_{2}$-nonconforming finite element space $\mathcal{N} C_{2}^{h}$.

Theorem 3.4. Let $\varphi_{j}^{i}, j=1, \cdots, N_{i}, i=E, V, Q$ be the functions defined in Definition 3.1. Either by omitting any one of vertex-based functions or any one of edge-based functions,

$$
\mathcal{B}_{1}=\left\{\varphi_{1}^{E}, \varphi_{2}^{E}, \cdots, \varphi_{N_{E}}^{E}, \varphi_{1}^{V}, \varphi_{2}^{V}, \cdots, \varphi_{N_{V}-1}^{V}, \varphi_{1}^{Q}, \varphi_{2}^{Q}, \cdots, \varphi_{N_{Q}}^{Q}\right\}
$$

or

$$
\mathcal{B}_{2}=\left\{\varphi_{1}^{E}, \varphi_{2}^{E}, \cdots, \varphi_{N_{E}-1}^{E}, \varphi_{1}^{V}, \varphi_{2}^{V}, \cdots, \varphi_{N_{V}}^{V}, \varphi_{1}^{Q}, \varphi_{2}^{Q}, \cdots, \varphi_{N_{Q}}^{Q}\right\}
$$

forms a set of global basis functions for $\mathcal{N C}_{2}^{h}$.

Proof. The proof of linear independence for the set $\mathcal{B}_{1}$ comes from a similar procedure as Theorem 2.6 in [41]. Indeed, if the linear combination for all vectors in $\mathcal{B}_{1}$ is zero, then the coefficients, say $c_{k}^{Q}$, related to $\varphi_{k}^{Q}$ are zero for all $k=1, \cdots, N_{Q}$, by the definition of bubble functions. To show that all the coefficients $c_{k}^{V}, c_{k}^{E}$ associated with $\varphi_{k}^{V}, \varphi_{k}^{E}$ are zero, suppose that $\mathbf{V}_{l}$ is a vertex adjacently connected to $\mathbf{V}_{N_{V}}$ by an edge $E_{k}=\overline{\mathbf{V}_{l} \mathbf{V}_{N_{V}}}$. By using the values of $\phi_{k}^{E}\left(\mathbf{G}_{j}\right)$ at the Gauss points $\mathbf{G}_{j} \in \mathcal{G}_{h}\left(N_{V}\right)$ that is also on $E_{k}$, one concludes that $c_{k}^{E}$ should vanish. This then leads to $c_{l}^{V}=0$ since $\mathbf{V}_{l}$ and $\mathbf{G}_{j}$ are connected via $E_{k}$. Since $\Omega$ is simply connected, by applying this sweeping out argument on edges and vertices that are connected to $\mathbf{V}_{l}$ 's, we can conclude that all $c_{k}^{V}, c_{k}^{E}$ are zeros. The dimension of the set $\mathcal{B}_{1}$ is $2 N_{E}$ which equals to the dimension of $\mathcal{N} C_{2}^{h}$. Thus, $\mathcal{B}_{1}$ forms a set of global basis functions for $\mathcal{N} C_{2}^{h}$. Similar arguments hold for the set $\mathcal{B}_{2}$.

Next, we investigate the dimension and a global basis function for $\mathcal{N} C_{2,0}^{h}$.

Theorem 3.5. Any function of $\mathcal{N} C_{2,0}^{h}$ can be written as the direct sum of a function in the piecewise $P_{2}$ conforming finite element space $X_{0, h}$ and one function in the bubble space $\Phi_{h}$, that is $\mathcal{N} C_{2,0}^{h}=X_{0, h} \oplus \Phi_{h}$. Moreover, $\operatorname{dim}\left(\mathcal{N} C_{2,0}^{h}\right)=N_{E}^{i}+N_{V}^{i}+N_{Q}=2 N_{E}^{i}+1$.

Proof. First, obviously $X_{0, h} \oplus \Phi_{h} \subset \mathcal{N} C_{2,0}^{h}$. Assume that there is a nontrivial function $\phi_{h} \in X_{0, h} \cap \Phi_{h}$ and apply the same argument as in the proof of Theorem 3.2. Then one sees that $\phi_{h}$ is identically zero in the whole domain $\Omega$ owing to the boundary condition. This contradicts to the assumption that $\phi_{h} \in X_{0, h} \cap \Phi_{h}$ is nontrivial. Therefore, $X_{0, h} \cap \Phi_{h}=\{0\}$. Thus, in order to prove the equality $X_{0, h} \oplus \Phi_{h}=\mathcal{N} C_{2,0}^{h}$, it is sufficient 
to prove $\operatorname{dim} \mathcal{N} C_{2,0}^{h}=\operatorname{dim} X_{0, h}+\operatorname{dim} \Phi_{h}$. The number of DOFs of $\mathcal{N} C_{2,0}^{h}$ is bounded by $2 N_{E}^{i}+1$ (see [41]). The number of DOFs of $X_{0, h} \oplus \Phi_{h}$ equals to $N_{E}^{i}+N_{V}^{i}+N_{Q}=2 N_{E}^{i}+1$, due to $N_{E}+N_{E}^{i}=4 N_{Q}, N_{E}^{i}+N_{V}=3 N_{Q}+1$ and $N_{E}^{b}=N_{V}^{b}$, where $N_{V}^{b}$ and $N_{E}^{b}$ are the number of boundary vertices and boundary edges, respectively. All together we have

$$
2 N_{E}^{i}+1=\operatorname{dim}\left(X_{0, h} \oplus \Phi_{h}\right) \leq \operatorname{dim} \mathcal{N} C_{2,0}^{h} \leq 2 N_{E}^{i}+1 .
$$

This completes the proof.

Theorem 3.6. $\mathcal{B}=\left\{\varphi_{1}^{E}, \varphi_{2}^{E}, \cdots, \varphi_{N_{E}^{i}}^{E}, \varphi_{1}^{V}, \varphi_{2}^{V}, \cdots, \varphi_{N_{V}^{i}}^{V}, \varphi_{1}^{Q}, \varphi_{2}^{Q}, \cdots, \varphi_{N_{Q}}^{Q}\right\}$ forms a set of global basis functions for $\mathcal{N} C_{2,0}^{h}$.

Proof. The proof of linear independence for $\mathcal{B}$ follows the same procedure in Theorem 3.4. The dimension of the set $\mathcal{B}$ is $2 N_{E}^{i}+1$ which equals to the dimension of $\mathcal{N} C_{2,0}^{h}$. Thus, $\mathcal{B}$ forms a set of global basis functions for $\mathcal{N} C_{2,0}^{h}$.

\section{THE ERROR ESTIMATES FOR ELLIPTIC PROBLEM}

In this section, we define some linear and interpolation operators and perform convergence analysis for elliptic problems with Robin boundary condition. Throughout the section, for an open bounded set $S \subset \mathbb{R}^{2}$ with its boundary $\partial S$, we will denote by $(\cdot, \cdot)_{S}$ and $\langle\cdot, \cdot\rangle_{\partial S}$ the $L^{2}(S)$ and $L^{2}(\partial S)$ inner products, respectively. If $S=\Omega$, these may be omitted from indices. For Sobolev spaces $H^{k}(S)$, their norms $\|\cdot\|_{H^{k}(S)}$ and seminorm $|\cdot|_{H^{k}(S)}$ are used.

\subsection{Some linear and interpolation operators}

Denote by $\gamma_{0}$ and $\gamma_{1}$ the trace maps from $H^{s+3 / 2}\left(Q_{j}\right)$ to $\Pi_{\mathbf{E} \subset \partial Q_{j}} H^{s}(\mathbf{E})$ such that $\gamma_{0} v=\left.v\right|_{\mathbf{E}}$ and $\gamma_{1} v=\left.\frac{\partial v}{\partial \nu}\right|_{\mathbf{E}}$. Then set

$$
\begin{aligned}
& \widetilde{\Lambda}^{h}=\Pi_{\mathbf{E} \in \mathcal{E}_{h}^{i}}\left[L^{2}(\mathbf{E}) \times L^{2}(\mathbf{E})\right] \times \Pi_{\mathbf{E} \in \mathcal{E}_{h}^{b}}, \\
& \Lambda^{h}=\left\{\lambda \in \Pi_{\mathbf{E} \in \mathcal{E}_{h}^{i}}\left[P_{1}(\mathbf{E}) \times P_{1}(\mathbf{E})\right] \times \Pi_{\mathbf{E} \in \mathcal{E}_{h}^{b}} P_{1}(\mathbf{E}): \lambda_{j}=\gamma_{0}\left(\left.\lambda\right|_{Q_{j}}\right) \in P_{1}(\mathbf{E}) ; \lambda_{j}+\lambda_{k}=0\right. \\
& \left.\forall \mathbf{E}=\partial Q_{j} \cap \partial Q_{k} ; \lambda_{j}=\gamma_{0}\left(\left.\lambda\right|_{Q_{j}}\right) \in P_{1}(\mathbf{E}) \forall \mathbf{E}=\partial Q_{j} \cap \partial \Omega\right\},
\end{aligned}
$$

where $P_{1}(\mathbf{E})$ denotes the set of linear functions on the edge $\mathbf{E}$. Denoting by $\mathcal{P}_{\mathbf{E}}^{1}: \widetilde{\Lambda}^{h} \rightarrow \Lambda^{h}$ the $L^{2}$ projection. Then the composition map $\left(\mathcal{P}_{\mathbf{E}}^{1} \circ \gamma_{1}\right): H^{3 / 2}(\Omega) \rightarrow \Lambda^{h}$ fulfills

$$
\left\langle\frac{\partial v_{j}}{\partial \boldsymbol{\nu}_{j}}-\left(\left(\mathcal{P}_{\mathbf{E}}^{1} \circ \gamma_{1}\right) v\right)_{j}, z\right\rangle_{\mathbf{E}}=0 \quad \text { for all } z \in P_{1}(\mathbf{E}) \text {, for all } \mathbf{E} \cap \partial Q_{j} \in \mathcal{E}_{h}^{i} \cup \mathcal{E}_{h}^{b},
$$

where $v_{j}=\left.v\right|_{Q_{j}}$ and $\boldsymbol{\nu}_{j}$ denotes the unit outward normal to $Q_{j}$. Then, from the standard polynomial approximation result we have

$$
\left\{\sum_{j=1}^{N_{Q}}\left\|\frac{\partial v_{j}}{\partial \boldsymbol{\nu}_{j}}-\left(\left(\mathcal{P}_{\mathbf{E}}^{1} \circ \gamma_{1}\right) v\right)_{j}\right\|_{L^{2}\left(\partial Q_{j}\right)}^{2}\right\}^{1 / 2} \leq C h^{s}\|v\|_{H^{s+3 / 2}(\Omega)}, \quad 1<s \leq 2 .
$$

Denote $\mathbf{E}_{j k}=\partial Q_{j} \cap \partial Q_{k}$ for all $Q_{j}, Q_{k} \in \mathcal{T}_{h}$ whenever the intersection is nonempty. Since $v_{j}-v_{k}$ has zero values at the Gauss points on $\mathbf{E}_{j k}$ for all $v \in \mathcal{N} C_{2}^{h}$ and the two points Gauss quadrature rule is exact up to polynomials of degree three, the following useful orthogonality holds.

Proposition 4.1. If $u \in H^{3 / 2}(\Omega)$, then the following orthogonality holds: for all $w \in \mathcal{N} C_{2}^{h}$,

$$
\left\langle\left(\left(\mathcal{P}_{\mathbf{E}}^{1} \circ \gamma_{1}\right) u\right)_{j}, w_{j}\right\rangle_{\mathbf{E}_{j k}}+\left\langle\left(\left(\mathcal{P}_{\mathbf{E}}^{1} \circ \gamma_{1}\right) u\right)_{k}, w_{k}\right\rangle_{\mathbf{E}_{k j}}=\left\langle\left(\left(\mathcal{P}_{\mathbf{E}}^{1} \circ \gamma_{1}\right) u\right)_{j}, w_{j}-w_{k}\right\rangle_{\mathbf{E}_{j k}}=0 .
$$


Furthermore, employing the following notation

$$
\gamma_{0}\left(\mathcal{N} C_{2}^{h}\right)=\left\{\Pi_{\mathbf{E}=\partial Q_{j} \cap \partial Q_{k} \in \mathcal{E}_{h}^{i}}\left(\left.\gamma_{0} w_{h}\right|_{\partial Q_{j} \cap \mathbf{E}},\left.\gamma_{0} w_{h}\right|_{\partial Q_{k} \cap \mathbf{E}}\right) \times\left.\Pi_{\mathbf{E} \in \mathcal{E}_{h}^{b}} \gamma_{0} w_{h}\right|_{\mathbf{E}} \forall w_{h} \in \mathcal{N} C_{2}^{h}\right\},
$$

which is a subset of $\widetilde{\Lambda}^{h}$, designate by $\Pi_{\mathbf{E}}^{1}: \gamma_{0}\left(\mathcal{N} C_{2}^{h}\right) \rightarrow \Lambda^{h}$ the interpolation such that $\Pi_{\mathbf{E}}^{1}\left(\gamma_{0} w_{h}\right)$ and $w_{h}$ coincide at the two Gauss points on $\mathbf{E}$ for every edge $\mathbf{E}$ in $\mathcal{T}_{h}$ for all $w_{h} \in \mathcal{N} C_{2}^{h}$. Then we have

$$
\sum_{Q \in \mathcal{T}_{h}} \sum_{\mathbf{E} \subset \partial Q \backslash \partial \Omega}\left\langle w_{h}-\left(\Pi_{\mathbf{E}}^{1} \circ \gamma_{0}\right)\left(w_{h}\right), z\right\rangle_{\mathbf{E}}=0 \forall z \in \Pi_{\mathbf{E} \in \mathcal{E}_{h}^{i}} P_{1}(\mathbf{E})
$$

The following orthogonality is also valid.

Proposition 4.2. If $u \in H^{3 / 2}(\Omega)$, then the following orthogonality holds:

$$
\sum_{Q \in \mathcal{T}_{h}} \sum_{\mathbf{E} \subset \partial Q \backslash \partial \Omega}\left\langle\frac{\partial u}{\partial \boldsymbol{\nu}_{\mathbf{E}}}-\left(\mathcal{P}_{\mathbf{E}}^{1} \circ \gamma_{1}\right) u,\left(\Pi_{\mathbf{E}}^{1} \circ \gamma_{0}\right)\left(w_{h}\right)\right\rangle_{\mathbf{E}}=0 \quad \text { for all } w \in \mathcal{N} C_{2}^{h},
$$

where $\boldsymbol{\nu}_{\mathbf{E}}$ denotes the unit outward normal to $\partial Q$.

Let $Q$ be a quadrilateral in $\mathcal{T}_{h}$ and $\widetilde{\Pi}_{Q}$ be a conforming interpolation operator $\widetilde{\Pi}_{Q}: H^{2}(Q) \rightarrow S_{2}^{1}\left(Q^{*}\right)$ defined by

$$
\begin{aligned}
\widetilde{\Pi}_{Q} \phi\left(\mathbf{V}_{j}\right) & =\phi\left(\mathbf{V}_{j}\right), \quad j=1, \cdots, 4, \\
\int_{\mathbf{E}_{j}} \widetilde{\Pi}_{Q} \phi \mathrm{d} \sigma & =\int_{\mathbf{E}_{j}} \phi \mathrm{d} \sigma, \quad j=1, \cdots, 4,
\end{aligned}
$$

for all $\phi \in H^{2}(Q)$. Then define the local interpolation operator $\Pi_{Q}: H^{2}(Q) \rightarrow S_{2}^{1}\left(Q^{*}\right)$ by

$$
\Pi_{Q} \phi=\widetilde{\Pi}_{Q} \phi+\alpha_{Q} \phi^{Q}
$$

so that the real number $\alpha_{Q}$ is chosen such that

$$
\Pi_{Q} \phi\left(\mathbf{O}_{Q}\right)=\phi\left(\mathbf{O}_{Q}\right)
$$

where $\mathbf{O}_{Q}$ denotes the intersection point of the two diagonals in $Q$. In other words, the interpolant $\Pi_{Q} \phi$ is a perturbation of $\widetilde{\Pi}_{Q} \phi$ by a bubble function $\phi^{Q}$. The global interpolation operator $\Pi_{h}: H^{2}(\Omega) \rightarrow \mathcal{N} C_{2}^{h}$ is then defined by localization.

Denote by $\|\cdot\|_{m, h}$ and $|\cdot|_{m, h}$ the usual mesh-dependent norm and seminorm:

$$
\|v\|_{m, h}=\left[\sum_{Q \in \mathcal{T}_{h}}\|v\|_{H^{m}(Q)}^{2}\right]^{1 / 2} ; \quad|v|_{m, h}=\left[\sum_{Q \in \mathcal{T}_{h}}|v|_{H^{m}(Q)}^{2}\right]^{1 / 2} .
$$

Since $\Pi_{Q}$ preserves $S_{2}^{1}\left(Q^{*}\right)$ for all $Q \in \mathcal{T}_{h}$ and $P_{2}(Q) \subset S_{2}^{1}\left(Q^{*}\right)$, it follows from the Bramble-Hilbert lemma [7,19] that

$$
\left\|\phi-\Pi_{h} \phi\right\|_{0, h}+h\left\|\phi-\Pi_{h} \phi\right\|_{1, h} \leq C h^{s}|\phi|_{H^{s}(\Omega)}, \quad \phi \in H^{s}(\Omega), 2<s \leq 3
$$




\subsection{A Robin boundary value problem}

We consider the following second-order elliptic problem with Robin boundary condition:

$$
\begin{aligned}
-\Delta u+\alpha u=f & \text { in } & \Omega, \\
\beta u+\frac{\partial u}{\partial \boldsymbol{\nu}}=g & \text { on } & \partial \Omega,
\end{aligned}
$$

where $\alpha \in L^{\infty}(\Omega)$ and $\beta \in L^{\infty}(\partial \Omega)$ are nonnegative, $f \in L^{2}(\Omega), g \in H^{1 / 2}(\partial \Omega)$.

The weak problem of (4.10) is then to find $u \in H^{1}(\Omega)$ such that

$$
a(u, v)=(f, v)+\langle g, v\rangle \quad \text { for all } \quad v \in H^{1}(\Omega),
$$

where the bilinear form $a(\cdot, \cdot): H^{1}(\Omega) \times H^{1}(\Omega) \rightarrow \mathbb{R}$ is given by

$$
a(u, v)=(\nabla u, \nabla v)+(\alpha u, v)+\langle\beta u, v\rangle, \quad u, v \in H^{1}(\Omega) .
$$

Also, the piecewise $P_{2}$-nonconforming finite element method is to find a solution $u_{h} \in \mathcal{N} C_{2}^{h}$ such that

$$
a_{h}\left(u_{h}, v_{h}\right)=\left(f, v_{h}\right)+\left\langle g, v_{h}\right\rangle, \quad v_{h} \in \mathcal{N} C_{2}^{h},
$$

where

$$
a_{h}\left(u_{h}, v_{h}\right)=\sum_{Q \in \mathcal{T}_{h}}\left(\nabla u_{h}, \nabla v_{h}\right)_{Q}+\left(\alpha u_{h}, v_{h}\right)+\left\langle\beta u_{h}, v_{h}\right\rangle, \quad u_{h}, v_{h} \in \mathcal{N} C_{2}^{h} .
$$

Since $\mathcal{N} C_{2}^{h}$ contains the conforming space $X_{h}$, we have the following orthogonality result:

$$
a_{h}\left(u-u_{h}, w_{h}\right)=0 \quad \text { for all } w_{h} \in X_{h} .
$$

\subsection{The error estimates}

To show an optimal order of convergence results, we first recall the well-known second Strang's lemma [53,54].

Lemma 4.3. Let $u \in H^{1}(\Omega)$ and $u_{h} \in \mathcal{N} C_{2}^{h}$ be the solutions of (4.11) and (4.12), respectively. Then, one has

$$
\left\|u-u_{h}\right\|_{1, h} \leq C\left\{\inf _{v_{h} \in \mathcal{N} C_{2}^{h}}\left\|u-v_{h}\right\|_{1, h}+\sup _{w_{h} \in \mathcal{N} C_{2}^{h}, w_{h} \neq 0} \frac{\left|a_{h}\left(u, w_{h}\right)-\left(f, w_{h}\right)-\left\langle g, w_{h}\right\rangle\right|}{\left\|w_{h}\right\|_{1, h}}\right\} .
$$

Assume sufficient regularity such that $u \in H^{3}(\Omega)$. Due to (4.9), the first term in the right side of (4.14) is bounded by

$$
\inf _{v_{h} \in \mathcal{N} C_{2}^{h}}\left\|u-v_{h}\right\|_{1, h} \leq\left\|u-\Pi_{h} u\right\|_{1, h} \leq C h^{s}|u|_{H^{s+1}(\Omega)}, 1<s \leq 2 .
$$

In order to bound the second term of the right side of (4.15) which denotes the consistency error, integrate by parts elementwise so that

$$
a_{h}\left(u, w_{h}\right)-\left(f, w_{h}\right)-\left\langle g, w_{h}\right\rangle=\sum_{Q \in \mathcal{T}_{h}}\left\langle\frac{\partial u}{\partial \boldsymbol{\nu}_{Q}}, w_{h}\right\rangle_{\partial Q \backslash \partial \Omega}, \quad w_{h} \in \mathcal{N} C_{2}^{h},
$$

where $\boldsymbol{\nu}_{Q}$ designates the unit outward normal vector to $\partial Q$.

We have the following lemma, which plays an important role in the analysis of nonconforming methods. 
Lemma 4.4. Let $u \in H^{s+1}(\Omega)$ for $1<s \leq 2$. Then we have the following estimate, for all $w_{h} \in \mathcal{N} C_{2}^{h}$,

$$
\left|\sum_{Q \in \mathcal{T}_{h}}\left\langle\frac{\partial u}{\partial \boldsymbol{\nu}_{Q}}, w_{h}\right\rangle_{\partial Q \backslash \partial \Omega}\right| \leq C h^{s}|u|_{H^{s+1}(\Omega)}\left\|w_{h}\right\|_{1, h}, 1<s \leq 2 .
$$

Proof. First, owing to (4.3), we have

$$
\sum_{Q \in \mathcal{T}_{h}}\left\langle\frac{\partial u}{\partial \boldsymbol{\nu}_{Q}}, w_{h}\right\rangle_{\partial Q \backslash \partial \Omega}=\sum_{Q \in \mathcal{T}_{h}} \sum_{\mathbf{E} \subset \partial Q \backslash \partial \Omega}\left\langle\frac{\partial u}{\partial \boldsymbol{\nu}_{\mathbf{E}}}-\left(\mathcal{P}_{\mathbf{E}}^{1} \circ \gamma_{1}\right) u, w_{h}\right\rangle_{\mathbf{E}} .
$$

Next, by using (4.5), (4.1), the trace theorem, and (4.2), it follows from (4.17) that

$$
\begin{aligned}
\left|\sum_{Q \in \mathcal{T}_{h}}\left\langle\frac{\partial u}{\partial \boldsymbol{\nu}_{Q}}, w_{h}\right\rangle_{\partial Q \backslash \partial \Omega}\right| & =\left|\sum_{Q \in \mathcal{T}_{h}} \sum_{\mathbf{E} \subset \partial Q \backslash \partial \Omega}\left\langle\frac{\partial u}{\partial \boldsymbol{\nu}_{\mathbf{E}}}-\left(\mathcal{P}_{\mathbf{E}}^{1} \circ \gamma_{1}\right) u, w_{h}-\left(\Pi_{\mathbf{E}}^{1} \circ \gamma_{0}\right)\left(w_{h}\right)\right\rangle_{\mathbf{E}}\right| \\
& \leq \sum_{Q \in \mathcal{T}_{h}} \sum_{\mathbf{E} \subset \partial Q \backslash \partial \Omega}\left\|\frac{\partial u}{\partial \boldsymbol{\nu}_{\mathbf{E}}}-\left(\mathcal{P}_{\mathbf{E}}^{1} \circ \gamma_{1}\right) u\right\|_{L^{2}(\mathbf{E})}\left\|w_{h}-\left(\Pi_{\mathbf{E}}^{1} \circ \gamma_{0}\right)\left(w_{h}\right)\right\|_{L^{2}(\mathbf{E})} \\
& \leq C h^{s} \sum_{Q \in \mathcal{T}_{h}}|u|_{H^{s+1}(Q)}\left\|w_{h}\right\|_{H^{1}(Q)} \\
& \leq C h^{s}|u|_{H^{s+1}(\Omega)}\left\|w_{h}\right\|_{1, h}, \text { for } 1<s \leq 2 .
\end{aligned}
$$

This proves the lemma.

Owing to Lemma 4.4 applied to (4.16), the consistency term is bounded as follows:

$$
\left|a_{h}\left(u, w_{h}\right)-\left(f, w_{h}\right)-\left\langle g, w_{h}\right\rangle\right| \leq C h^{s}|u|_{H^{s+1}(\Omega)}\left\|w_{h}\right\|_{1, h}, 1<s \leq 2 .
$$

A combination of (4.15) and (4.18) leads to a discrete $H^{1}$-norm error estimate, summarized in the following theorem.

Theorem 4.5. Let $u \in H^{s+1}(\Omega), 1<s \leq 2$, and $u_{h} \in \mathcal{N} C_{2}^{h}$ be the solutions of (4.11) and (4.12), respectively. Then we have

$$
\left\|u-u_{h}\right\|_{1, h} \leq C h^{s}|u|_{H^{s+1}(\Omega)}, 1<s \leq 2 .
$$

Next, in order to derive an $L^{2}$-error estimate, we use the Aubin-Nitsche duality argument [41]. Set $e_{h}=u-u_{h}$ and let $\eta \in H^{2}(\Omega)$ be the solution of the dual problem:

$$
\begin{array}{ccc}
-\Delta \eta+\alpha \eta=e_{h} & \text { in } & \Omega, \\
\beta \eta+\frac{\partial \eta}{\partial \boldsymbol{\nu}}=0 & \text { on } & \partial \Omega .
\end{array}
$$

with the elliptic regularity:

$$
\|\eta\|_{H^{2}(\Omega)} \leq C\left\|e_{h}\right\|_{L^{2}(\Omega)} .
$$

Let $w_{h} \in L^{2}(\Omega)$ be arbitrary such that

$$
\left.w_{h}\right|_{Q} \in H^{1}(Q) \forall Q \in \mathcal{T}_{h} \text { and }\left.\int_{\mathbf{E}_{j k}} w_{h}\right|_{Q_{j}} \mathrm{~d} \sigma=\left.\int_{\mathbf{E}_{k j}} w_{h}\right|_{Q_{k}} \mathrm{~d} \sigma, \quad Q_{j}, Q_{k} \in \mathcal{T}_{h},
$$


where $\mathbf{E}_{j k}=\mathbf{E}_{k j}=\partial Q_{j} \cap \partial Q_{k}$. Then, by a similar argument as in the previous consistency error estimate, we have

$$
\left|a_{h}\left(w_{h}, \eta\right)-\left(w_{h}, e_{h}\right)\right| \leq C h\|\eta\|_{H^{2}(\Omega)}\left\|w_{h}\right\|_{1, h} .
$$

In particular, with the choice $w_{h}=e_{h}$,

$$
\left|a_{h}\left(e_{h}, \eta\right)-\left(e_{h}, e_{h}\right)\right| \leq C h\|\eta\|_{H^{2}(\Omega)}\left\|e_{h}\right\|_{1, h} .
$$

Let $\eta_{h}$ be the conforming interpolant of $\eta$ to $\mathcal{N} C_{2}^{h}$ as in (4.6). Then, from (4.13), we get the orthogonality:

$$
a_{h}\left(e_{h}, \eta_{h}\right)=0 .
$$

Now, from (4.19)-(4.21) and Theorem 4.5 it follows that

$$
\begin{aligned}
\left\|e_{h}\right\|_{L^{2}(\Omega)}^{2} & \leq\left|a_{h}\left(e_{h}, \eta-\eta_{h}\right)\right|+C h\|\eta\|_{H^{2}(\Omega)}\left\|e_{h}\right\|_{1, h} \\
& \leq C\left\|\eta-\eta_{h}\right\|_{H^{1}(\Omega)}\left\|e_{h}\right\|_{1, h}+C h\|\eta\|_{H^{2}(\Omega)}\left\|e_{h}\right\|_{1, h} \\
& \leq C h\|\eta\|_{H^{2}(\Omega)}\left\|e_{h}\right\|_{1, h} \\
& \leq C h\left\|e_{h}\right\|_{L^{2}(\Omega)}\left\|e_{h}\right\|_{1, h} \\
& \leq C h^{s+1}\left\|e_{h}\right\|_{L^{2}(\Omega)}|u|_{H^{s+1}(\Omega)}, 1<s \leq 2 .
\end{aligned}
$$

Summarizing the above, we have the following $L^{2}$-error estimate:

Theorem 4.6. Let $u \in H^{s+1}(\Omega), 1<s \leq 2$, and $u_{h} \in \mathcal{N} C_{2}^{h}$ be the solutions of (4.11) and (4.12), respectively. Then we have

$$
\left\|u-u_{h}\right\|_{L^{2}(\Omega)} \leq C h^{s+1}|u|_{H^{s+1}(\Omega)}, 1<s \leq 2 .
$$

\section{The ERror estimates For the Stokes Problem}

In this section, we will apply the proposed piecewise $P_{2}$-nonconforming element for the approximation of each component of velocity and the piecewise $P_{1}$-nonconforming element for the pressure to solve the Stokes problem. We will derive an optimal order error estimate in broken energy norm.

\subsection{The Stokes problem}

Consider the following stationary Stokes equations:

$$
\begin{aligned}
&-\mu \Delta \mathbf{u}+\nabla p=\mathbf{f} \text { in } \quad \Omega, \\
& \operatorname{div} \mathbf{u}=0 \quad \text { in } \quad \Omega, \\
& \mathbf{u}=\mathbf{0} \quad \text { on } \quad \partial \Omega,
\end{aligned}
$$

where $\mathbf{u}=\left(u_{1}, u_{2}\right)^{T}$ represents the velocity vector, $p$ the pressure, $\mathbf{f}=\left(f_{1}, f_{2}\right)^{T}$ the body force, and $\mu>0$ denotes the viscosity. As usual set

$$
L_{0}^{2}(\Omega)=\left\{q \in L^{2}(\Omega) \mid \int_{\Omega} q \mathrm{~d} \mathbf{x}=0\right\}
$$

and consider the variational formulation of (5.1) to seek a pair $(\mathbf{u}, p) \in\left[H_{0}^{1}(\Omega)\right]^{2} \times L_{0}^{2}(\Omega)$ such that

$$
\begin{aligned}
& a(\mathbf{u}, \mathbf{v})-b(\mathbf{v}, p)=(\mathbf{f}, \mathbf{v}) \quad \forall \quad \mathbf{v} \in\left[H_{0}^{1}(\Omega)\right]^{2}, \\
& b(\mathbf{u}, q)=0 \quad \forall \quad q \in L_{0}^{2}(\Omega),
\end{aligned}
$$


where the bilinear forms are defined by

$$
a(\mathbf{u}, \mathbf{v})=\mu(\nabla \mathbf{u}, \nabla \mathbf{v})=\mu \sum_{j=1}^{2}\left(\nabla u_{j}, \nabla v_{j}\right) \quad \text { and } \quad b(\mathbf{v}, q)=(\operatorname{div} \mathbf{v}, q) .
$$

Assume that the domain is sufficiently smooth so that the solution of $(5.2)$ is $H^{s}(\Omega)$-regular for $1<s \leq 2$. In other words, for any $\mathbf{f} \in\left[H^{s-2}(\Omega)\right]^{2}$, the Stokes problem has a unique solution $\mathbf{u} \in\left[H^{s}(\Omega)\right]^{2} \cap\left[H_{0}^{1}(\Omega)\right]^{2}$ and $p \in L_{0}^{2}(\Omega) \cap H^{s-1}(\Omega)$ satisfying the following a priori estimate:

$$
\|\mathbf{u}\|_{H^{s}(\Omega)}+\|p\|_{H^{s-1}(\Omega)} \leq C\|\mathbf{f}\|_{H^{s-2}(\Omega)},
$$

where $C$ is a constant independent of the $\mathbf{f}$.

Set $\mathbf{V}_{h}=\left[\mathcal{N} C_{2,0}^{h}\right]^{2}$ and by $W_{h}$ designate the space of piecewise $P_{1}$-nonconforming quadrilateral element [46]:

$$
W_{h}=\left\{q \in L_{0}^{2}(\Omega)|q|_{Q_{j}} \in P_{1}\left(Q_{j}\right) \quad \forall Q_{j} \in \mathcal{T}_{h}\right\} .
$$

Then, the nonconforming method is to find $\left(\mathbf{u}_{h}, p_{h}\right) \in \mathbf{V}_{h} \times W_{h}$ fulfilling

$$
\begin{aligned}
a_{h}\left(\mathbf{u}_{h}, \mathbf{v}_{h}\right)-b_{h}\left(\mathbf{v}_{h}, p_{h}\right) & =\left(\mathbf{f}, \mathbf{v}_{h}\right) & & \forall \mathbf{v}_{h} \in \mathbf{V}_{h}, \\
b_{h}\left(\mathbf{u}_{h}, q_{h}\right) & =0 & & \forall q_{h} \in W_{h},
\end{aligned}
$$

where $a_{h}(\mathbf{u}, \mathbf{v})=\mu \sum_{Q \in \mathcal{T}_{h}}(\nabla \mathbf{u}, \nabla \mathbf{v})_{Q}$ and $b_{h}(\mathbf{v}, q)=\sum_{Q \in \mathcal{T}_{h}}(\operatorname{div} \mathbf{v}, q)_{Q}$.

\subsection{The linear maps and interpolation operators}

The linear maps and interpolation operators defined for elliptic problems are extended componentwise to the vector-valued case, and will be used to analyze convergence of the nonconforming solutions.

Let $\mathbf{P}_{\mathbf{E}}^{1} \circ \gamma_{1}:\left[H^{3 / 2}(\Omega)\right]^{2} \rightarrow\left[\Lambda^{h}\right]^{2}$ and $\left(\boldsymbol{\Pi}_{\mathbf{E}}^{1} \circ \gamma_{0}\right): \mathbf{V}_{h} \rightarrow\left[\Pi_{\mathbf{E} \in \mathcal{E}_{h}} P_{1}(\mathbf{E})\right]^{2}$ be defined such that their components are defined by $\mathcal{P}_{\mathbf{E}}^{1} \circ \gamma_{1}$ and $\Pi_{\mathbf{E}}^{1} \circ \gamma_{0}$ as in Section 4.1 and recall (4.1)-(4.3) and (4.4)-(4.5) for each vector component, respectively. Also define the projections $\mathcal{P}_{W_{h}}: L^{2}(\Omega) \rightarrow W_{h}$ and $\mathcal{P}_{\mathbf{E}}^{0}: L^{2}(\mathbf{E}) \rightarrow P_{0}(\mathbf{E})$ for each edge $\mathbf{E}$ by

$$
\begin{aligned}
\left(\mathcal{P}_{W_{h}} q, \eta\right)_{Q_{j}} & =(q, \eta)_{Q_{j}} \quad \forall \eta \in P_{1}\left(Q_{j}\right) \text { and } \forall Q_{j} \in \mathcal{T}_{h}, \forall q \in L^{2}(\Omega), \\
\left\langle\mathcal{P}_{\mathbf{E}}^{0} q, \zeta\right\rangle_{\mathbf{E}} & =\langle q, \zeta\rangle_{\mathbf{E}} \quad \forall \zeta \in P_{0}(\mathbf{E}), \forall q \in L^{2}(\mathbf{E}) .
\end{aligned}
$$

It then follows from the standard polynomial approximation result that

$$
\begin{gathered}
\left\|\mathcal{P}_{W_{h}} q-q\right\|_{0, h}+h\left|\mathcal{P}_{W_{h}} q-q\right|_{1, h} \leq C h^{2}\|q\|_{H^{2}(\Omega)} \quad \forall q \in \Pi_{Q_{j} \in \mathcal{T}_{h}} H^{2}\left(Q_{j}\right), \\
\left\{\sum_{\partial Q_{j}}\left\|\mathcal{P}_{\mathbf{E}}^{0} q-q\right\|_{L^{2}\left(\partial Q_{j}\right)}^{2}\right\}^{1 / 2} \leq C h\|q\|_{H^{3 / 2}\left(Q_{j}\right)} \quad \forall q \in H^{3 / 2}\left(Q_{j}\right) .
\end{gathered}
$$

The following lemma will be useful.

Lemma 5.1. Let $\mathbf{w} \in\left[H^{s+1}(\Omega)\right]^{2}, 1<s \leq 2$, and $q \in H^{2}(\Omega)$. Then we have the following estimates:

$$
\begin{aligned}
& \left|\sum_{Q \in \mathcal{T}_{h}} \sum_{\mathbf{E} \subset \partial Q \backslash \partial \Omega}\left\langle\frac{\partial \mathbf{w}}{\partial \boldsymbol{\nu}}, \mathbf{v}\right\rangle_{\mathbf{E}}\right| \leq C h^{s}\|\mathbf{w}\|_{H^{s+1}(\Omega)}|\mathbf{v}|_{1, h} \forall \mathbf{v} \in\left[H_{0}^{1}(\Omega)\right]^{2} \cup \mathbf{V}_{h}, \\
& \left|\sum_{Q \in \mathcal{T}_{h}} \sum_{\mathbf{E} \subset \partial Q \backslash \partial \Omega}\left\langle q, \boldsymbol{\nu} \cdot \mathbf{w}_{h}\right\rangle_{\mathbf{E}}\right| \leq C h^{2}\|q\|_{H^{2}(\Omega)}\left|\mathbf{w}_{h}\right|_{1, h} \quad \forall \mathbf{w}_{h} \in \mathbf{V}_{h} .
\end{aligned}
$$


Proof. Let $\mathbf{v} \in\left[H_{0}^{1}(\Omega)\right]^{2} \cup \mathbf{V}_{h}$ be arbitrary. The estimate (5.7a) is obvious from Lemma 4.4. Next, let $q \in H^{2}(\Omega)$ be given. By using the linear map $\Pi_{\mathbf{E}}^{1} \circ \gamma_{0}$ and the trace theorem, we have

$$
\begin{aligned}
\left|\sum_{Q \in \mathcal{T}_{h}} \sum_{\mathbf{E} \subset \partial Q \backslash \partial \Omega}\left\langle q, \boldsymbol{\nu} \cdot \mathbf{w}_{h}\right\rangle_{\mathbf{E}}\right| & =\left|\sum_{Q \in \mathcal{T}_{h}} \sum_{\mathbf{E} \subset \partial Q \backslash \partial \Omega}\left\langle q-\left(\Pi_{\mathbf{E}}^{1} \circ \gamma_{0}\right) q, \boldsymbol{\nu} \cdot \mathbf{w}_{h}\right\rangle_{\mathbf{E}}\right| \\
& =\left|\sum_{Q \in \mathcal{T}_{h}} \sum_{\mathbf{E} \subset \partial Q \backslash \partial \Omega}\left\langle q-\left(\Pi_{\mathbf{E}}^{1} \circ \gamma_{0}\right) q, \boldsymbol{\nu} \cdot\left(\mathbf{w}_{h}-\mathbf{P}_{\mathbf{E}}^{0} \mathbf{w}_{h}\right)\right\rangle_{\mathbf{E}}\right| \\
& \leq C h^{3 / 2}\|q\|_{H^{2}(\Omega)} h^{1 / 2}\left|\mathbf{w}_{h}\right|_{1, h}=C h^{2}\|q\|_{H^{2}(\Omega)}\left|\mathbf{w}_{h}\right|_{1, h},
\end{aligned}
$$

where $\mathbf{P}_{\mathbf{E}}^{0}$ is the componentwise extension of $\mathcal{P}_{\mathbf{E}}^{0}$ to vectors. Hence, the proof is complete.

We defined the interpolation operator $\Pi_{h}$ for the elliptic problems in Section 4. In this subsection, we also introduce the interpolation operator $\boldsymbol{\Pi}_{h}$ so that it satisfies the hypothesis H.1 of Crouzeix and Raviart [21], that is

$$
\begin{aligned}
\boldsymbol{\Pi}_{h} & \in \mathcal{L}\left(\left[H^{2}(\Omega)\right]^{2} ; \mathbf{V}_{h}\right), \\
\left(\operatorname{div} \boldsymbol{\Pi}_{h} \mathbf{v}, q_{h}\right) & =\left(\operatorname{div} \mathbf{v}, q_{h}\right) \quad \forall q_{h} \in W_{h}, \\
\left\|\mathbf{v}-\boldsymbol{\Pi}_{h} \mathbf{v}\right\|_{1, h} & \leq C h^{2}|\mathbf{v}|_{H^{3}(\Omega)} \quad \forall \mathbf{v} \in\left[H^{3}(\Omega)\right]^{2} .
\end{aligned}
$$

Now, we consider the interpolation operator $\boldsymbol{\Pi}_{Q}$ in two steps. We first define for $\mathbf{v}=\left(v_{1}, v_{2}\right)^{T} \in\left[H^{2}(Q)\right]^{2}$,

$$
\widetilde{\Pi}_{Q} \mathbf{v}=\left(\begin{array}{c}
\widetilde{\Pi}_{Q} v_{1} \\
\widetilde{\Pi}_{Q} v_{2}
\end{array}\right)
$$

where $\widetilde{\Pi}_{Q} v_{i}$ is defined by relations (4.6). We thereafter define

$$
\boldsymbol{\Pi}_{Q} \mathbf{v}=\widetilde{\boldsymbol{\Pi}}_{Q} \mathbf{v}+\left(\begin{array}{c}
\alpha_{Q}^{1} \phi^{Q}(\mathbf{x}) \\
\alpha_{Q}^{2} \phi^{Q}(\mathbf{x})
\end{array}\right)
$$

where $\alpha_{Q}^{1}$ and $\alpha_{Q}^{2}$ are determined such that

$$
\int_{Q} \operatorname{div} \boldsymbol{\Pi}_{Q} \mathbf{v} q_{h} \mathrm{~d} \mathbf{x}=\int_{Q} \operatorname{div} \mathbf{v} q_{h} \mathrm{~d} \mathbf{x} \quad \forall q_{h} \in P_{1}(Q)
$$

The global interpolation operator $\boldsymbol{\Pi}_{h}:\left[H^{2}(\Omega)\right]^{2} \rightarrow \mathbf{V}_{h}$ is then extended by using $\boldsymbol{\Pi}_{Q}$. The analogue of (4.9) holds:

$$
\left\|\mathbf{v}-\Pi_{h} \mathbf{v}\right\|_{0, h}+h\left\|\mathbf{v}-\Pi_{h} \mathbf{v}\right\|_{1, h} \leq C h^{s}|\mathbf{v}|_{H^{s}(\Omega)}, \quad \mathbf{v} \in H^{s}(\Omega), 2<s \leq 3 .
$$

\subsection{The inf-sup condition and error estimates}

It is well-known that the bilinear form $b(\cdot, \cdot)$ satisfies the continuous inf-sup condition, i.e., there exists a positive constant $\beta$ such that

$$
\sup _{\mathbf{v} \in\left[H_{0}^{1}(\Omega)\right]^{2}} \frac{b(\mathbf{v}, q)}{|\mathbf{v}|_{1, \Omega}} \geq \beta\|q\|_{L^{2}(\Omega)} \quad \forall q \in L_{0}^{2}(\Omega) .
$$


The argument in references $[13,17,21]$ proves that the bilinear form $b_{h}(\cdot, \cdot)$ satisfies a discrete inf-sup condition on the pair of the finite element space $\mathbf{V}_{h} \times W_{h}$ such that, for any $q_{h} \in W_{h} \subset L_{0}^{2}(\Omega)$,

$$
\begin{aligned}
\sup _{\mathbf{v}_{h} \in \mathbf{V}_{h}} \frac{b_{h}\left(\mathbf{v}_{h}, q_{h}\right)}{\left|\mathbf{v}_{h}\right|_{1, h}} & \geq \sup _{\mathbf{w} \in\left[H^{2}(\Omega)\right]^{2}} \frac{b_{h}\left(\boldsymbol{\Pi}_{h} \mathbf{w}, q_{h}\right)}{\left|\boldsymbol{\Pi}_{h} \mathbf{w}\right|_{1, h}} \\
& =\sup _{\mathbf{w} \in\left[H^{2}(\Omega)\right]^{2}} \frac{b\left(\mathbf{w}, q_{h}\right)}{|\mathbf{w}|_{1, h}} \\
& \geq \beta\left\|q_{h}\right\|_{L^{2}(\Omega)} .
\end{aligned}
$$

Moreover, in this subsection, we introduce the optimal-order error estimates in the (broken) energy-norm for the velocity and the $L^{2}$-norm for the pressure. The energy-norm error analysis in the velocity is based on (5.2) and (5.4), and then an application of the discrete inf-sup condition (5.13) estimate results in the error estimate of the pressure.

Lemma 5.2. Let $(\mathbf{u}, p) \in\left[H^{3}(\Omega)\right]^{2} \times H^{2}(\Omega)$ and $\left(\mathbf{u}_{h}, p\right) \in \mathbf{V}_{h} \times W_{h}$ be the solutions of (5.2) and (5.4), respectively. Then the following estimates hold:

$$
\begin{gathered}
\left|\mathbf{u}-\mathbf{u}_{h}\right|_{1, h} \leq \inf _{\mathbf{v}_{h} \in \mathbf{V}_{h}}\left|\mathbf{u}-\mathbf{v}_{h}\right|_{1, h}+\sup _{\mathbf{v}_{h} \in \mathbf{V}_{h}} \frac{\left|a_{h}\left(\mathbf{u}, \mathbf{v}_{h}\right)-b_{h}\left(\mathbf{v}_{h}, p\right)-\left(\mathbf{f}, \mathbf{v}_{h}\right)\right|}{\left|\mathbf{v}_{h}\right|_{1, h}} \\
\left\|p-p_{h}\right\|_{L^{2}(\Omega)} \leq \inf _{q_{h} \in W_{h}}\left|p-q_{h}\right|_{L^{2}(\Omega)}+\sup _{\mathbf{v}_{h} \in \mathbf{V}_{h}} \frac{\left|a_{h}\left(\mathbf{u}, \mathbf{v}_{h}\right)-b_{h}\left(\mathbf{v}_{h}, p\right)-\left(\mathbf{f}, \mathbf{v}_{h}\right)\right|}{\left|\mathbf{v}_{h}\right|_{1, h}} \\
\quad+\inf _{\mathbf{v}_{h} \in \mathbf{V}_{h}}\left|\mathbf{u}-\mathbf{v}_{h}\right|_{1, h} .
\end{gathered}
$$

Proof. For $\mathbf{v}_{h} \in \mathbf{V}_{h}$, if follows from (5.2) and (5.4) that

$$
\begin{aligned}
\mu\left|\mathbf{u}_{h}-\mathbf{v}_{h}\right|_{1, h}^{2} & =a_{h}\left(\mathbf{u}_{h}-\mathbf{v}_{h}, \mathbf{u}_{h}-\mathbf{v}_{h}\right) \\
& =a_{h}\left(\mathbf{u}_{h}, \mathbf{u}_{h}-\mathbf{v}_{h}\right)-a_{h}\left(\mathbf{u}, \mathbf{u}_{h}-\mathbf{v}_{h}\right)+a_{h}\left(\mathbf{u}-\mathbf{v}_{h}, \mathbf{u}_{h}-\mathbf{v}_{h}\right) \\
& =\left(\mathbf{f}, \mathbf{u}_{h}-\mathbf{v}_{h}\right)+b_{h}\left(\mathbf{u}_{h}-\mathbf{v}_{h}, p\right)-a_{h}\left(\mathbf{u}, \mathbf{u}_{h}-\mathbf{v}_{h}\right)+a_{h}\left(\mathbf{u}-\mathbf{v}_{h}, \mathbf{u}_{h}-\mathbf{v}_{h}\right) .
\end{aligned}
$$

Dividing both sides of (5.15) by $\left|\mathbf{u}_{h}-\mathbf{v}_{h}\right|_{1, h}$ gives

$$
\mu\left|\mathbf{u}_{h}-\mathbf{v}_{h}\right|_{1, h} \leq \sup _{\mathbf{w}_{h} \in \mathbf{V}_{h}} \frac{\left|\left(\mathbf{f}, \mathbf{w}_{h}\right)+b_{h}\left(\mathbf{w}_{h}, p\right)-a_{h}\left(\mathbf{u}, \mathbf{w}_{h}\right)\right|}{\left|\mathbf{w}_{h}\right|_{1, h}}+\left|\mathbf{u}-\mathbf{v}_{h}\right|_{1, h} .
$$

By using the triangle inequality and (5.16), we see that

$$
\left|\mathbf{u}-\mathbf{u}_{h}\right|_{1, h} \leq \frac{1}{\mu} \sup _{\mathbf{w}_{h} \in \mathbf{V}_{h}} \frac{\left|\left(\mathbf{f}, \mathbf{w}_{h}\right)+b_{h}\left(\mathbf{w}_{h}, p\right)-a_{h}\left(\mathbf{u}, \mathbf{w}_{h}\right)\right|}{\left|\mathbf{w}_{h}\right|_{1, h}}+\left(1+\frac{1}{\mu}\right) \inf _{\mathbf{v}_{h} \in \mathbf{V}_{h}}\left|\mathbf{u}-\mathbf{v}_{h}\right|_{1, h},
$$

which proves $(5.14 \mathrm{a})$.

Next, for $\mathbf{v}_{h} \in \mathbf{V}_{h}$ and $q_{h} \in W_{h}$, from the discrete variational formulation (5.4), we get

$$
\begin{aligned}
b_{h}\left(\mathbf{v}_{h}, q_{h}-p_{h}\right) & =b_{h}\left(\mathbf{v}_{h}, q_{h}-p\right)+b_{h}\left(\mathbf{v}_{h}, p\right)-b_{h}\left(\mathbf{v}_{h}, p_{h}\right) \\
& =b_{h}\left(\mathbf{v}_{h}, q_{h}-p\right)+b_{h}\left(\mathbf{v}_{h}, p\right)+\left(\mathbf{f}, \mathbf{v}_{h}\right)-a_{h}\left(\mathbf{u}_{h}, \mathbf{v}_{h}\right) \\
& =b_{h}\left(\mathbf{v}_{h}, q_{h}-p\right)+a_{h}\left(\mathbf{u}-\mathbf{u}_{h}, \mathbf{v}_{h}\right)+\left[\left(\mathbf{f}, \mathbf{v}_{h}\right)+b_{h}\left(\mathbf{v}_{h}, p\right)-a_{h}\left(\mathbf{u}, \mathbf{v}_{h}\right)\right] .
\end{aligned}
$$

It thus follows from the above equality that

$$
\begin{aligned}
\sup _{\mathbf{v}_{h} \in \mathbf{V}_{h}} \frac{\left|b_{h}\left(\mathbf{v}_{h}, q_{h}-p_{h}\right)\right|}{\left|\mathbf{v}_{h}\right|_{1, h}} \leq & C\left\{\| q_{h}-p||_{L^{2}(\Omega)}+\left|\mathbf{u}-\mathbf{u}_{h}\right|_{1, h}\right\} \\
& +\sup _{\mathbf{v}_{h} \in \mathbf{V}_{h}} \frac{\left|\left(\mathbf{f}, \mathbf{v}_{h}\right)+b_{h}\left(\mathbf{v}_{h}, p\right)-a_{h}\left(\mathbf{u}, \mathbf{v}_{h}\right)\right|}{\left|\mathbf{v}_{h}\right|_{1, h}}
\end{aligned}
$$


By using the triangle inequality, we have

$$
\left\|p-p_{h}\right\|_{L^{2}(\Omega)} \leq\left\|p-q_{h}\right\|_{L^{2}(\Omega)}+\left\|p_{h}-q_{h}\right\|_{L^{2}(\Omega)} .
$$

From the discrete the inf-sup condition (5.13), we have

$$
\beta\left\|p_{h}-q_{h}\right\|_{L^{2}(\Omega)} \leq \sup _{\mathbf{v}_{h} \in \mathbf{V}_{h}} \frac{\left|b_{h}\left(\mathbf{v}_{h}, q_{h}-p_{h}\right)\right|}{\left|\mathbf{v}_{h}\right|_{1, h}}
$$

Then, a combination of the above inequalities (5.21)-(5.23) and result of (5.14a) leads to (5.14c).

Theorem 5.3. Let $(\mathbf{u}, p) \in\left[H^{3}(\Omega)\right]^{2} \times H^{2}(\Omega)$ and $\left(\mathbf{u}_{h}, p\right) \in \mathbf{V}_{h} \times W_{h}$ be the solutions of (5.2) and (5.4), respectively. Then there exists a positive constant $C$ such that

$$
\left|\mathbf{u}-\mathbf{u}_{h}\right|_{1, h}+\left\|p-p_{h}\right\|_{L^{2}(\Omega)} \leq C h^{2}\left(\|\mathbf{u}\|_{H^{3}(\Omega)}+\|p\|_{H^{2}(\Omega)}\right) .
$$

Proof. Multiply (5.1) by $\mathbf{v}_{h} \in \mathbf{V}_{h}$, and integrating by parts on each element, we see that

$$
\begin{aligned}
\left(\mathbf{f}, \mathbf{v}_{h}\right) & =\left(-\mu \Delta \mathbf{u}+\nabla p, \mathbf{v}_{h}\right) \\
& =a_{h}\left(\mathbf{u}, \mathbf{v}_{h}\right)-b_{h}\left(\mathbf{v}_{h}, p\right)-\mu \sum_{Q \in \mathcal{T}_{h}} \sum_{\mathbf{E} \subset \partial Q \backslash \partial \Omega}\left\langle\frac{\partial \mathbf{u}}{\partial \boldsymbol{\nu}}, \mathbf{v}_{h}\right\rangle_{\mathbf{E}}+\sum_{Q \in \mathcal{T}_{h}} \sum_{\mathbf{E} \subset \partial Q \backslash \partial \Omega}\left\langle p, \boldsymbol{\nu} \cdot \mathbf{v}_{h}\right\rangle_{\mathbf{E}} .
\end{aligned}
$$

By using the Lemma 5.1, we get

$$
\begin{aligned}
\left|\left(\mathbf{f}, \mathbf{v}_{h}\right)-a_{h}\left(\mathbf{u}, \mathbf{v}_{h}\right)+b_{h}\left(\mathbf{v}_{h}, p\right)\right| & \leq \mu\left|\sum_{Q \in \mathcal{T}_{h}} \sum_{\mathbf{E} \subset \partial Q \backslash \partial \Omega}\left\langle\frac{\partial \mathbf{u}}{\partial \boldsymbol{\nu}}, \mathbf{v}_{h}\right\rangle_{\mathbf{E}}\right|+\left|\sum_{Q \in \mathcal{T}_{h}} \sum_{\mathbf{E} \subset \partial Q \backslash \partial \Omega}\left\langle p, \nu \cdot \mathbf{v}_{h}\right\rangle_{\mathbf{E}}\right| \\
& \leq C_{1} h^{2}\left(\|\mathbf{u}\|_{H^{3}(\Omega)}+\|p\|_{H^{2}(\Omega)}\right)\left|\mathbf{v}_{h}\right|_{1, h},
\end{aligned}
$$

where $C_{1}$ is constant. From (5.25) and Lemma 5.2, we obtain

$$
\begin{aligned}
\left|\mathbf{u}-\mathbf{u}_{h}\right|_{1, h}+\left\|p-p_{h}\right\|_{L^{2}(\Omega)} \leq & \inf _{\mathbf{v}_{h} \in \mathbf{V}_{h}}\left|\mathbf{u}-\mathbf{v}_{h}\right|_{1, h} \\
& +\inf _{q_{h} \in W_{h}}|| p-q_{h} \|_{L^{2}(\Omega)}+\sup _{\mathbf{v}_{h} \in \mathbf{V}_{h}} \frac{\left|a_{h}\left(\mathbf{u}, \mathbf{v}_{h}\right)-b_{h}\left(\mathbf{v}_{h}, p\right)-\left(\mathbf{f}, \mathbf{v}_{h}\right)\right|}{\left|\mathbf{v}_{h}\right|_{1, h}} \\
\leq & \inf _{\mathbf{v}_{h} \in \mathbf{V}_{h}}\left|\mathbf{u}-\mathbf{v}_{h}\right|_{1, h}+\inf _{q_{h} \in W_{h}}\left\|p-q_{h}\right\|_{L^{2}(\Omega)} \\
& +C_{1} h^{2}\left(\|\mathbf{u}\|_{H^{3}(\Omega)}+\|p\|_{H^{2}(\Omega)}\right) .
\end{aligned}
$$

We choose $\mathbf{v}_{h}:=\boldsymbol{\Pi}_{h} \mathbf{v} \in \mathbf{V}_{h}$ and $q_{h}:=\mathcal{P}_{W_{h}} q \in W_{h}$, for $\mathbf{v} \in\left[H^{3}(\Omega)\right]^{2}$ and $q \in H^{2}(\Omega)$. By using (5.6) and (5.12), we can have the estimates (5.24). This completes the proof of the theorem.

\section{NumericAl RESUltS}

In this section, we describe our numerical algorithms and their applications to the Robin, Neumann, and Dirichlet elliptic problems by using the proposed piecewise $P_{2}$-nonconforming finite element. In addition, we illustrate our numerical algorithms and their applications to the Stokes problem. 
TABLE 1. The Robin problem: The apparent $L^{2}$ and broken energy norm errors and their reduction ratios on the quadrilateral meshes.

\begin{tabular}{|l|c|c|c|c|c|}
\hline$h$ & DOFs & $\left\|u-u_{h}\right\|_{0}$ & ratio & $\left\|u-u_{h}\right\|_{h}$ & ratio \\
\hline $1 / 4$ & 80 & $0.108139 \mathrm{E}-01$ & - & $0.368472 \mathrm{E}+00$ & - \\
$1 / 8$ & 288 & $0.174690 \mathrm{E}-02$ & 2.63 & $0.108033 \mathrm{E}+00$ & 1.77 \\
$1 / 16$ & 1088 & $0.234655 \mathrm{E}-03$ & 2.90 & $0.275774 \mathrm{E}-01$ & 1.97 \\
$1 / 32$ & 4224 & $0.320626 \mathrm{E}-04$ & 2.87 & $0.720034 \mathrm{E}-02$ & 1.94 \\
$1 / 64$ & 16640 & $0.400834 \mathrm{E}-05$ & 3.00 & $0.179782 \mathrm{E}-02$ & 2.00 \\
$1 / 128$ & 66048 & $0.500288 \mathrm{E}-06$ & 3.00 & $0.448901 \mathrm{E}-03$ & 2.00 \\
\hline
\end{tabular}

\subsection{Numerical implementation}

Let $\Omega$ be a unit square. In order to generate a quadrilateral mesh, we first generate a uniform quadrilateral mesh, and then perturb it randomly for each vertex (see Fig. 7). We solve the discrete bilinear forms for the Robin, Neumann, and Dirichlet boundary problems and the stationary Stokes problem. In order to check error decay behavior precisely, our numerical integration to calculate the discretized weak form (4.12) adopts a 24point quadrature rule for each quadrilateral [20]. In our implementation, it is necessary to physically construct the four subtriangles of each quadrilateral. Indeed, each quadrilateral $Q$ is first decomposed into four triangles by its diagonals $\ell_{13}$ and $\ell_{24}$ as shown in Figure 1 . Then for each triangle, we choose the quadrature rule based on six barycentric points which are exact upto polynomials of degree four. If, instead, we use simply the four point or the nine point Gauss quadrature rule on each quadrilateral, we are not able to get sufficiently precise point values in numerical integration that contain the polynomials $\left(\ell_{13}^{+}\right)^{2}$ and $\left(\ell_{24}^{+}\right)^{2}$.

\subsection{Numerical examples for elliptic problems}

In this subsection, we illustrate three numerical examples of elliptic problem. After assembling the mass and stiffness matrices, one arrives at the linear system $A x=b$ where $A$ is a symmetric, positive definite matrix. The linear system is solved by the CG (Conjugate Gradient) method with initial guess $x_{0}=0.0$ and tolerance $\epsilon=10^{-10}$.

First, consider the Robin problem:

$$
\begin{aligned}
& -\Delta u+u=f \quad \text { in } \quad \Omega, \\
& u+\frac{\partial u}{\partial \nu}=g \quad \text { on } \quad \partial \Omega,
\end{aligned}
$$

where $\Omega=(0,1)^{2}$. The source terms $f$ and $g$ are generated from the exact solution

$$
u(x, y)=\cos (2 \pi x) \cos (2 \pi y)\left(x^{3}-y^{4}+x^{2} y^{3}\right) .
$$

Table 1 shows the numerical results on the quadrilateral meshes using the proposed piecewise $P_{2}$-nonconforming finite element, where the error reduction ratios in $L^{2}$ and energy norms are optimal. The generated quadrilateral mesh for $16 \times 16$ case is shown in Figure 7 .

As a second example, consider the Neumann problem:

$$
\begin{aligned}
& -\Delta u+u=f \quad \text { in } \quad \Omega, \\
& \frac{\partial u}{\partial \nu}=g \quad \text { on } \quad \partial \Omega,
\end{aligned}
$$

where $\Omega=(0,1)^{2}$. The source terms $f$ and $g$ are generated from the same exact solution used in Robin problem. Table 2 shows the numerical results on the meshes using the proposed piecewise $P_{2}$-nonconforming finite element. 
TABLE 2. The Neumann problem: The apparent $L^{2}$ and broken energy norm errors and their reduction ratios on the quadrilateral meshes.

\begin{tabular}{|l|c|c|c|c|c|}
\hline$h$ & DOFs & $\left\|u-u_{h}\right\|_{0}$ & ratio & $\left\|u-u_{h}\right\|_{h}$ & ratio \\
\hline $1 / 4$ & 80 & $0.110603 \mathrm{E}-01$ & - & $0.367487 \mathrm{E}+00$ & - \\
$1 / 8$ & 288 & $0.175355 \mathrm{E}-02$ & 2.66 & $0.107900 \mathrm{E}+00$ & 1.77 \\
$1 / 16$ & 1088 & $0.234800 \mathrm{E}-03$ & 2.90 & $0.275582 \mathrm{E}-01$ & 1.97 \\
$1 / 32$ & 4224 & $0.320650 \mathrm{E}-04$ & 2.87 & $0.719831 \mathrm{E}-02$ & 1.94 \\
$1 / 64$ & 16640 & $0.400822 \mathrm{E}-05$ & 3.00 & $0.179756 \mathrm{E}-02$ & 2.00 \\
$1 / 128$ & 66048 & $0.500270 \mathrm{E}-06$ & 3.00 & $0.448870 \mathrm{E}-03$ & 2.00 \\
\hline
\end{tabular}

TABLE 3. The Dirichlet problem: The apparent $L^{2}$ and broken energy norm errors and their reduction ratios on the quadrilateral meshes.

\begin{tabular}{|l|c|c|c|c|c|}
\hline$h$ & DOFs & $\left\|u-u_{h}\right\|_{0}$ & ratio & $\left\|u-u_{h}\right\|_{h}$ & ratio \\
\hline $1 / 4$ & 49 & $0.102292 \mathrm{E}-01$ & - & $0.358321 \mathrm{E}+00$ & - \\
$1 / 8$ & 225 & $0.176445 \mathrm{E}-02$ & 2.54 & $0.107652 \mathrm{E}+00$ & 1.73 \\
$1 / 16$ & 961 & $0.240300 \mathrm{E}-03$ & 2.88 & $0.282108 \mathrm{E}-01$ & 1.93 \\
$1 / 32$ & 3369 & $0.318292 \mathrm{E}-04$ & 2.92 & $0.753306 \mathrm{E}-02$ & 1.90 \\
$1 / 64$ & 16129 & $0.403027 \mathrm{E}-05$ & 2.98 & $0.187971 \mathrm{E}-02$ & 2.00 \\
$1 / 128$ & 65025 & $0.503134 \mathrm{E}-06$ & 3.00 & $0.470513 \mathrm{E}-03$ & 2.00 \\
\hline
\end{tabular}

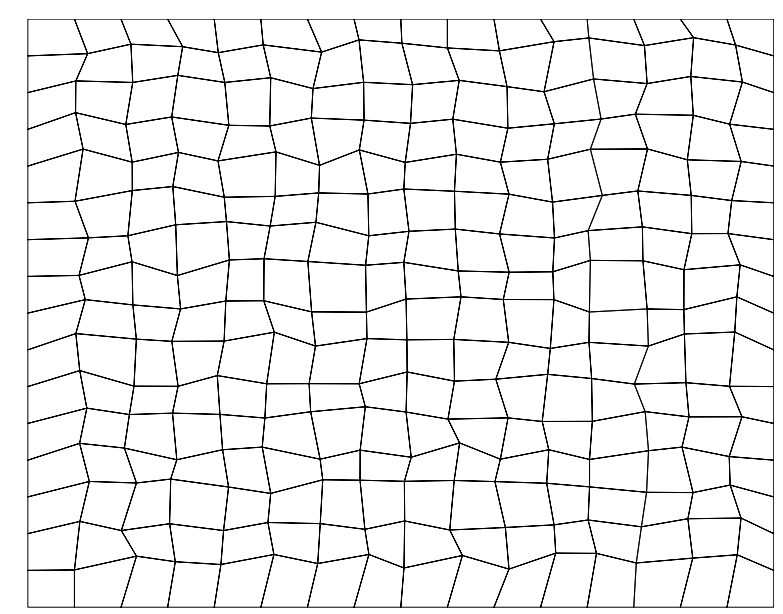

FiguRE 7 . The generated quadrilateral mesh for $16 \times 16$ case.

Finally, we consider the following Dirichlet problem:

$$
\begin{array}{rll}
-\Delta u+u=f & \text { in } \quad & \Omega, \\
u=0 & \text { on } & \partial \Omega,
\end{array}
$$

where $\Omega=(0,1)^{2}$. The source term $f$ is calculated from the exact solution

$$
u(x, y)=\sin (2 \pi x) \sin (2 \pi y)\left(x^{3}-y^{4}+x^{2} y^{3}\right) .
$$

Table 3 shows the numerical results on the quadrilateral meshes using the proposed piecewise $P_{2}$-nonconforming finite element. The error reduction ratios in $L^{2}$ and energy norm are optimal. 
TABLE 4. The Stokes problem: The apparent $L^{2}$ and broken energy norm errors and their reduction ratios on the quadrilateral meshes.

\begin{tabular}{|l|c|c|c|c|c|c|c|}
\hline$h$ & DOFs & $\left\|u-u_{h}\right\|_{0}$ & ratio & $\left|u-u_{h}\right|_{1}$ & ratio & $\left\|p-p_{h}\right\|_{0}$ & ratio \\
\hline $1 / 4$ & 106 & $0.129588 \mathrm{E}-01$ & - & $0.166315 \mathrm{E}+00$ & - & $0.212764 \mathrm{E}+00$ & - \\
$1 / 8$ & 498 & $0.105722 \mathrm{E}-02$ & 3.62 & $0.342813 \mathrm{E}-01$ & 2.28 & $0.422747 \mathrm{E}-01$ & 2.33 \\
$1 / 16$ & 2146 & $0.129835 \mathrm{E}-03$ & 3.03 & $0.824496 \mathrm{E}-02$ & 2.06 & $0.954216 \mathrm{E}-02$ & 2.15 \\
$1 / 32$ & 8898 & $0.160508 \mathrm{E}-04$ & 3.02 & $0.212246 \mathrm{E}-02$ & 1.96 & $0.232163 \mathrm{E}-02$ & 2.04 \\
$1 / 64$ & 36226 & $0.211149 \mathrm{E}-05$ & 2.93 & $0.536311 \mathrm{E}-03$ & 1.98 & $0.567958 \mathrm{E}-03$ & 2.03 \\
\hline
\end{tabular}

\subsection{Numerical examples for the Stokes problem}

In this subsection, we present an example in the two dimensional Stokes problem to illustrate the validity of the theoretical results obtained in the previous section.

The velocity and pressure variables are approximated by using randomly generated quadrilateral meshes.

First, the exact solution for $\mathbf{u}$, which is divergence-free, is given by $\nabla \times \psi$, where

$$
\psi(x, y)=\exp (x+2 y) x^{2}(x-1)^{2} y^{2}(y-1)^{2},
$$

with the exact solution for $p$ given by

$$
-\sin (2 \pi x) \sin (2 \pi y) .
$$

Then the body force term $\mathbf{f}$ can be generated by $-\Delta \mathbf{u}+\nabla p$. The numerical results are presented in Table 4 in terms of the $H^{1}$-norm and $L^{2}$-norm convergence rates. Also, in this table, DOFs mean the number of degrees of freedom for the velocity and pressure. In our case, DOFs are explicitly given by $2 N_{E}^{i}+3 N_{V}^{i}+2 N_{Q}-1$.

\section{Conclusions}

In this paper, we have developed a piecewise $P_{2}$-nonconforming finite element method that can be used on genuinely quadrilateral meshes. We provide rigorous mathematical analysis about the DOFs and error estimates. We have confirmed that our numerical results match very well with theoretical results in the elliptic and Stokes problems. In addition, our proposed method can be extended further for other problems, such as the NavierStokes and elasticity problems. An extension to three dimensions is our on-going project.

Acknowledgements. This work has been supported in part by an international cooperative research agreement between Chinese NSFC and Korean NRF. This research was partially supported by NRF of Korea (Nos. 2011-0000344, F01-2009000-10122-0). This research is also supported by NNSFC (Nos. 109111440268, 10771028, 10801023, 10826071) and the Fundamental Research Funds for the Central Universities.

\section{REFERENCES}

[1] R. Altmann and C. Carstensen, $p_{1}$-nonconforming finite elements on triangulations into triangles and quadrilaterals. SIAM J. Numer. Anal. 50 (2012) 418-438.

[2] D.N. Arnold, F. Brezzi and M. Fortin, A stable finite element for the Stokes equations. Calcolo 21 (1984) $337-344$.

[3] D. N. Arnold and R. Winther, Nonconforming mixed elements for elasticity. Dedicated to Jim Douglas, Jr. on the occasion of his 75th birthday. Math. Models Methods Appl. Sci. 13 (2003) 295-307.

[4] I. Babuška and M. Suri, Locking effect in the finite element approximation of elasticity problem. Numer. Math. 62 (1992) 439-463.

[5] I. Babuška and M. Suri, On locking and robustness in the finie element method. SIAM J. Numer. Anal. 29 (1992) $1261-1293$.

[6] R. Bank and B. Welfert, A comparison between the mini-element and the Petrov-Galerkin formulations for the generalized Stokes problem. Comput. Methods Appl. Mech. Eng. 83 (1990) 61-68.

[7] J.H. Bramble and S.R. Hilbert, Estimation of linear functionals on Sobolev spaces with application to Fourier transforms and spline interpolation. SIAM J. Numer. Anal. 7 (1970) 113-124. 
[8] S. Brenner and L. Scott, The Mathematical Theorey of Finite Element Methods. Springer-Verlag, New York (1994).

[9] S.C. Brenner and L.Y. Sung, Linear finite element methods for planar elasticity. Math. Comput. 59 (1992) 321-338.

[10] F. Brezzi, M.-O. Bristeau, L.P. Franca, M. Mallet and G. Rogé, A relationship between stabilized finite element methods and the Galerkin method with bubble functions. Comput. Meth. Appl. Mech. Eng. 96 (1992) 117-129.

[11] F. Brezzi, A. Buffa and K. Lipnikov, Mimetic finite differences for elliptic problems. ESAIM-Math. Model. Numer. Anal. 43 (2009) 277-295.

[12] F. Brezzi and J. Douglas, Jr. Stabilized mixed methods for the Stokes problem. Numer. Math. 53 (1988) $225-236$.

[13] F. Brezzi and M. Fortin, Mixed and Hybrid Finite Element Methods, Springer-Verlag, New York. Springer Series Comput. Math. 15 (1991).

[14] F. Brezzi, K. Lipnikov and M. Shashkov, Convergence of the mimetic finite difference method for diffusion problems on polyhedral meshes. SIAM J. Numer. Anal. (2006) 1872-1896.

[15] A.N. Brooks and T.J.R. Hughes, Streamline upwind Petrov-Galerkin formulations for convective dominated flows with particular emphasis on the incompressible Navier-Stokes equations. Comput. Methods Appl. Mech. Eng. 32 (1982) $199-259$.

[16] Z. Cai, J. Douglas, Jr., J.E. Santos, D. Sheen and X. Ye, Nonconforming quadrilateral finite elements: A correction. Calcolo 37 (2000) 253-254.

[17] Z. Cai, J. Douglas, Jr. and X. Ye, A stable nonconforming quadrilateral finite element method for the stationary Stokes and Navier-Stokes equations. Calcolo 36 (1999) 215-232.

[18] C. Carstensen and J. Hu, A unifying theory of a posteriori error control for nonconforming finite element methods. Numer. Math. 107 (2007) 473-502.

[19] P.G. Ciarlet, The Finite Element Method for Elliptic Equations. North-Holland, Amsterdam (1978).

[20] G.R. Cowper, Gaussian quadrature formulas for triangles. Int. J. Num. Meth. Eng. 7 (1973) 405-408.

[21] M. Crouzeix and P.-A. Raviart. Conforming and nonconforming finite element methods for solving the stationary Stokes equations. RAIRO Math. Model. Anal. Numer. R-3 (1973) 33-75.

[22] L.B. da Veiga, V. Gyrya, K. Lipnikov and G. Manzini, Mimetic finite difference method for the Stokes problem on polygonal meshes. J. Comp. Phys. 228 (2009) 7215-7232.

[23] L.B. da Veiga, K. Lipnikov and G. Manzini, Convergence analysis of the high-order mimetic finite difference method. Numer. Math. 113 (2009) 325-356.

[24] L.B. da Veiga and G. Manzini, A higher-order formulation of the mimetic finite difference method. SIAM J. Sci. Comput. 31 (2008) 732-760.

[25] J. Douglas, Jr., J.E. Santos, D. Sheen and X. Ye, Nonconforming Galerkin methods based on quadrilateral elements for second order elliptic problems. ESAIM Math. Model. Numer. Anal. 33 (1999) 747-770.

[26] J. Douglas, Jr. and J. Wang. An absolutely stabilized finite element method for the Stokes problem. Math. Comput. 52 (1989) 495-508.

[27] R.S. Falk, Nonconforming finite element methods for the equations of linear elasticity. Math. Comput. 57 (1991) 529-550.

[28] M. Farhloul and M. Fortin, A mixed nonconforming finite element for the elasticity and Stokes problems. Math. Models Methods Appl. Sci. 9 (1999) 1179-1199.

[29] M. Fortin, A three-dimensional quadratic nonconforming element. Numer. Math. 46 (1985) 269-279.

[30] M. Fortin and M. Soulie, A non-conforming piecewise quadratic finite element on the triangle. Int. J. Numer. Meth. Eng. 19 (1983) 505-520.

[31] L. Franca, S. Frey and T. Hughes, Stabilized finite element methods: I. Application to the advective-diffusive model. Comput. Methods Appl. Mech. Eng. 95 (1992) 221-242.

[32] V. Girault and P.-A. Raviart, Finite Element Methods for Navier-Stokes Equations, Theory and Algorithms. Springer-Verlag, Berlin (1986).

[33] V. Gyrya and K. Lipnikov, High-order mimetic finite difference method for diffusion problems on polygonal meshes. J. Comput. Phys. 227 (2008) 8841-8854.

[34] H. Han, Nonconforming elements in the mixed finite element method. J. Comput. Math. 2 (1984) 223-233.

[35] P. Hood and C. Taylor, A numerical solution for the Navier-Stokes equations using the finite element technique. Computers Fluids 1 (1973) 73-100.

[36] T.J.R. Hughes and A.N. Brooks, A multidimensional upwind scheme with no crosswind diffusion, in Finite Element Methods for Convection Dominated Flows, edited by T.J.R. Hughes. ASME, New York (1979) 19-35.

[37] B.M. Irons and A. Razzaque, Experience with the patch test for convergence of finite elements, in The Mathematics of Foundation of the Finite Element Methods with Applications to Partial Differential Equations, edited by A.K. Aziz. Academic Press, New York (1972) 557-587.

[38] P. Klouček, B. Li and M. Luskin, Analysis of a class of nonconforming finite elements for crystalline microstructures. Math. Comput. 65 (1996) 1111-1135.

[39] M. Köster, A. Quazzi, F. Schieweck, S. Turek and P. Zajac, New robust nonconforming finite elements of higher order. Appl. Numer. Math. 62 (2012) 166-184.

[40] C.-O. Lee, J. Lee and D. Sheen, A locking-free nonconforming finite element method for planar elasticity. Adv. Comput. Math. 19 (2003) 277-291.

[41] H. Lee and D. Sheen, A new quadratic nonconforming finite element on rectangles. Numer. Methods Partial Differ. Equ. 22 (2006) 954-970. 
[42] P. Lesaint, On the convergence of Wilson's nonconforming element for solving the elastic problem. Comput. Methods Appl. Mech. Eng. 7 (1976) 1-76.

[43] B. Li and M. Luskin, Nonconforming finite element approximation of crystalline microstructure. Math. Comput. 67 (1998) 917-946.

[44] Z.X. Luo, Z.L. Meng and C.M. Liu, Computational Geometry - Theory and Applications of Surface Representation. Sinica Academic Press, Beijing (2010).

[45] P. Ming and Z.-C. Shi, Nonconforming rotated $Q_{1}$ element for Reissner-Mindlin plate. Math. Models Methods Appl. Sci. 11 (2001) 1311-1342.

[46] C. Park and D. Sheen. $P_{1}$-nonconforming quadrilateral finite element methods for second-order elliptic problems. SIAM J. Numer. Anal. 41 (2003) 624-640.

[47] R. Pierre, Simple $C^{0}$ approximations for the computation of incompressible flows. Comput. Methods Appl. Mech. Eng. 68 (1988) 205-227.

[48] R. Pierre, Regularization procedures of mixed finite element approximations of the Stokes problem. Numer. Methods Partial Differ. Equ. 5 (1989) 241-258.

[49] R. Rannacher and S. Turek. Simple nonconforming quadrilateral Stokes element. Numer. Methods Partial Differ. Equ. 8 (1992) 97-111.

[50] G. Sander and P. Beckers, The influence of the choice of connectors in the finite element method. Int. J. Numer. Methods Eng. 11 (1977) 1491-1505.

[51] Z.-C. Shi, A convergence condition for the quadrilateral Wilson element. Numer. Math. 44 (1984) 349-361.

[52] Z.-C. Shi, On the convergence properties of the quadrilateral elements of Sander and Beckers. Math. Comput. 42 (1984) 493-504.

[53] G. Strang, Variational crimes in the finite element method, in The Mathematical Foundations of the Finite Element Method with Applications to Partial Differential Equations, edited by A.K. Aziz. New York, Academic Press (1972) 689-710.

[54] G. Strang and G.J. Fix, An Analysis of the Finite Element Method. Prentice-Hall, Englewood Cliffs (1973).

[55] R. Wang, Multivariate Spline Functions and Their Applications. Science Press, Kluwer Academic Publishers (1994).

[56] E. L. Wilson, R. L. Taylor, W. P. Doherty and J. Ghaboussi, Incompatible displacement models, in Numerical and Computer Method in Structural Mechanics, Academic Press, New York (1973) 43-57.

[57] Z. Zhang, Analysis of some quadrilateral nonconforming elements for incompressible elasticity. SIAM J. Numer. Anal. 34 (1997) 640-663. 